\title{
MID-INFRARED VARIABILITY OF PROTOSTARS IN IC 1396A
}

\author{
M. Morales-Calderón ${ }^{1}$, J. R. Stauffer ${ }^{2}$, L. Rebull ${ }^{2}$, B. A. Whitney ${ }^{3}$, D. Barrado y Navascués ${ }^{1}$, D. R. Ardila ${ }^{2}$, I. Song $^{4}$, \\ T. Y. BrookE ${ }^{4}$, L. HartmanN ${ }^{5}$, AND N. CALVET ${ }^{5}$ \\ ${ }^{1}$ Laboratorio de Astrofísica Estelar y Exoplanetas (LAEX), Centro de Astrobiología (CAB, INTA-CSIC), LAEFF, P.O. 78, E-28691, Villanueva de la canada, \\ Madrid, Spain; mariamc@laeff.inta.es \\ ${ }^{2}$ Spitzer Science Center, California Institute of Technology, Pasadena, CA 91125, USA \\ ${ }^{3}$ Space Science Institute, 4750 Walnut Street, Suite 205, Boulder, CO 80301, USA \\ ${ }^{4}$ Astronomy Department, MC 105-24, California Institute of Technology, Pasadena, CA 91125, USA \\ ${ }^{5}$ Department of Astronomy, University of Michigan, 500 Church Street, Ann Arbor, MI 48109, USA \\ Received 2008 October 30; accepted 2009 July 21; published 2009 August 21
}

\begin{abstract}
We have used Spitzer/Infrared Array Camera (IRAC) to conduct a photometric monitoring program of the IC1396A dark globule in order to study the mid-IR $(3.6-8 \mu \mathrm{m})$ variability of the heavily embedded young stellar objects (YSOs) present in that area. We obtained light curves covering a 14 day timespan with a twice daily cadence for 69 YSOs, and continuous light curves with approximately $12 \mathrm{~s}$ cadence over $7 \mathrm{hr}$ for 38 YSOs. Typical accuracies for our relative photometry were $1 \%-2 \%$ for the long timespan data and a few millimagnitude, corresponding to less than $0.5 \%$, for the $7 \mathrm{hr}$ continuous "staring-mode" data. More than half of the YSOs showed detectable variability, with amplitudes from $\sim 0.05$ mag to $\sim 0.2$ mag. About $30 \%$ of the YSOs showed quasisinusoidal light-curve shapes with apparent periods from 5 to 12 days and light-curve amplitudes approximately independent of wavelength over the IRAC bandpasses. We have constructed models which simulate the timedependent spectral energy distributions of Class I and II YSOs in order to attempt to explain these light curves. Based on these models, the apparently periodic light curves are best explained by YSO models where one or two high-latitude photospheric spots heat the inner wall of the circumstellar disk, and where we view the disk at fairly large inclination angle. Disk inhomogeneities, such as increasing the height where the accretion funnel flows to the stellar hot spot, enhances the light-curve modulations. The other YSOs in our sample show a range of light-curve shapes, some of which are probably due to varying accretion rate or disk shadowing events. One star, IC1396A-47, shows a $3.5 \mathrm{hr}$ periodic light curve; this object may be a PMS Delta Scuti star.
\end{abstract}

Key words: infrared: stars - stars: pre-main sequence - stars: variables: other

Online-only material: color figures, machine-readable table

\section{INTRODUCTION}

IC1396A (aka "The Elephant Trunk Nebula") is a prominent dark globule seen projected onto the bright nebular emission of the HII region IC1396. IC1396 is itself part of the larger Cepheus OB2 association, which includes young clusters with ages ranging from 10-12 Myr (NGC 7160), to $4 \mathrm{Myr}(\operatorname{Tr} 37)$, to $<1 \mathrm{Myr}$ (the proto-clusters forming in globules such as IC1396A and IC1396N). Reach et al. (2004) reported the first Spitzer observations of IC1396A, where they used Infrared Array Camera (IRAC) to identify three Class 0 or I protostars and a dozen Class II young stellar objects (YSOs) within the boundaries of the globule. Sicilia-Aguilar et al. (2006) reported the results of a much wider area Spitzer IRAC and Multiband Imaging Photometer for Spitzer (MIPS) survey of the Cepheus OB2 association, where they derived disk frequencies and spectral energy distribution (SED) shapes for stars in Tr 37, NGC 7160, and IC1396A. Sicilia-Aguilar et al. identified more than 50 YSOs in IC1396A, including 11 Class I stars and 32 Class II YSOs. They suspected that a number of other YSOs were present in the field of view (FOV), but they could not be confirmed either because there was too much nebular contamination of the photometry or because their observations did not allow them to identify Class III sources (YSOs lacking IR excesses).

Most of the IC1396A YSO population is heavily extincted, so there is relatively little literature describing this proto-cluster. Because it is embedded in the IC1396 H II region, the normally assumed distance to the globule is that adopted for IC1396 and $\operatorname{Tr} 37$, which is $900 \mathrm{pc}$ (Contreras et al. 2002). Sicilia-Aguilar et al. compared optical photometry and spectral type data for six of the most lightly reddened globule members to Siess et al. (2000) isochrones, and concluded that these IC1396A stars have an average age of $\sim 1 \mathrm{Myr}$. The more heavily embedded members could presumably be even younger.

We have obtained time-series monitoring of the YSOs in IC1396A using Spitzer's IRAC camera in order to (1) study the temporal variability of these very young, heavily embedded stars and (2) identify additional members of the globule population. In Section 2, we describe the observations and our photometric data analysis. In Section 3, we provide new, deeper maps of the globule based on co-adding all of our observations and from these images construct a new list of YSO members of the globule population. Our time-series photometry and identification of variable stars is described in Section 4, while Section 5 includes a discussion of the different kinds of variability found and possible physical mechanisms to explain the variability. Finally, we summarize our findings in Section 6.

\section{OBSERVATIONS}

The goal of this program was to obtain well-sampled photometry for the protostars in IC1396A on timescales both of hours and days. Several previous programs have demonstrated that IRAC can provide extremely accurate and stable time-series photometry through the detection of the thermal emission of 


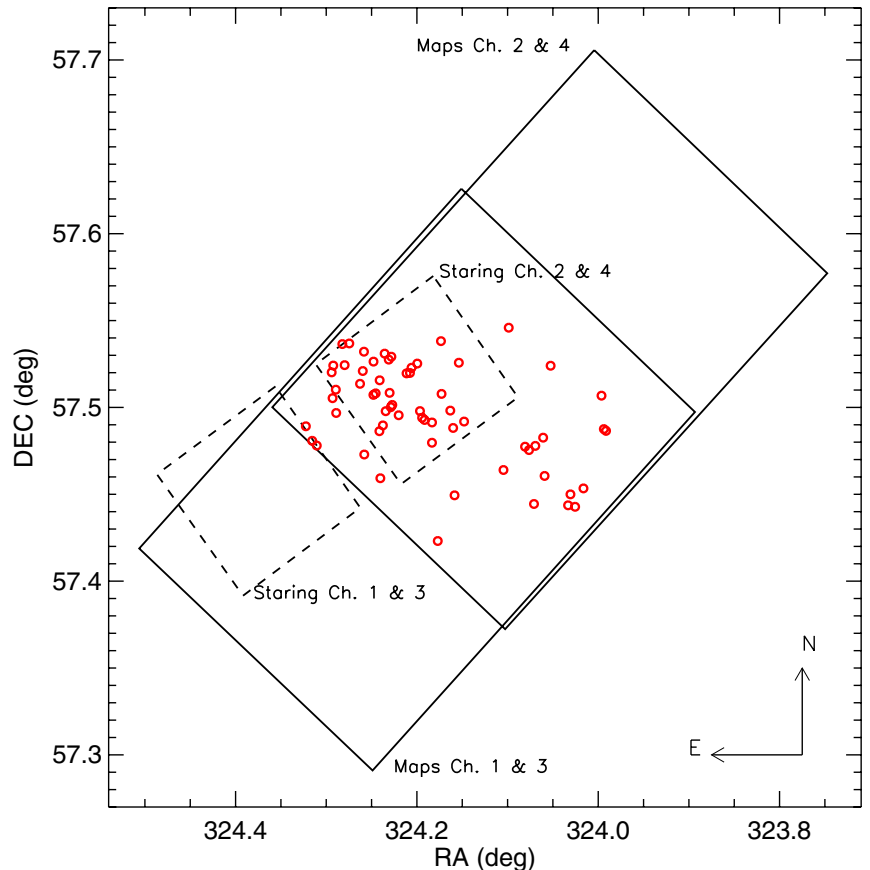

Figure 1. Layout of observations. YSOs from Sicilia-Aguilar et al. (2006) are marked with open circles. East is left, north is up. The solid and dashed rectangles show the FOV of the map and staring data, respectively. All the previously known members are located within the area where mapping observations at Ch. 1 and 3 and Ch. 2 and 4 overlap.

(A color version of this figure is available in the online journal.)

extra-solar planets (Charbonneau et al. 2005; Deming et al. 2007) and the attempt to detect cloud formations in the photospheres of brown dwarfs (Morales-Calderón et al. 2006). Thus, we have used a recent DDT program (PI: Soifer, PID:470) to study mid-IR variability of protostars in the very young starforming globule IC1396A. The data were collected from 2008 January 24 to February 6 and observations were performed with two distinctly different observing modes.

1. Mapping mode. IRAC imaging was obtained for the whole IC1396A globule in mapping mode. About every $12 \mathrm{hr}$ for 14 days, a short AOR was run to make a $2 \times 3$ map with individual exposures of $12 \mathrm{~s}$ frametime (corresponding to $10.4 \mathrm{~s}$ exposure times) and a five position dithering at each map step. The IRAC maps do not cover the same FOV in all bands, providing a region of $\sim 10^{\prime} \times 11.5$, centered at 21:36:30.85+57:29:49.37 with photometry in the four IRAC bands (see Figure 1). Fifty two YSOs from SiciliaAguilar et al. (2006) and 13 YSOs from Reach et al. (2004; 11 of which are in common with the sample from SiciliaAguilar et al.) are included in this region.

2. Staring mode. In addition, on February 6, a long staring AOR was performed. This AOR consisted of $\sim 7.5 \mathrm{hr}$ of continuous monitoring with no dithering or mapping to get a continuous set of photometry for a $\sim 5^{\prime} \times 5^{\prime}$ region. A total of 2000 single observations, of $12 \mathrm{~s}$ frametime each, were performed. The Ch. 2 and Ch. 4 FOV was positioned at the core of the globule and it included 37 previously known YSOs. The center of the Ch. 1 and Ch. 3 FOV is offset from the Ch. 2 and Ch. 4 FOV by of order $7^{\prime}$ to the SE. No previously known YSOs fall in that region. See Figure 1 for further information on the layout of observations.

We will use the staring data (Ch. 2 and Ch. 4) to study the short-term (from minutes to hours) variability and the
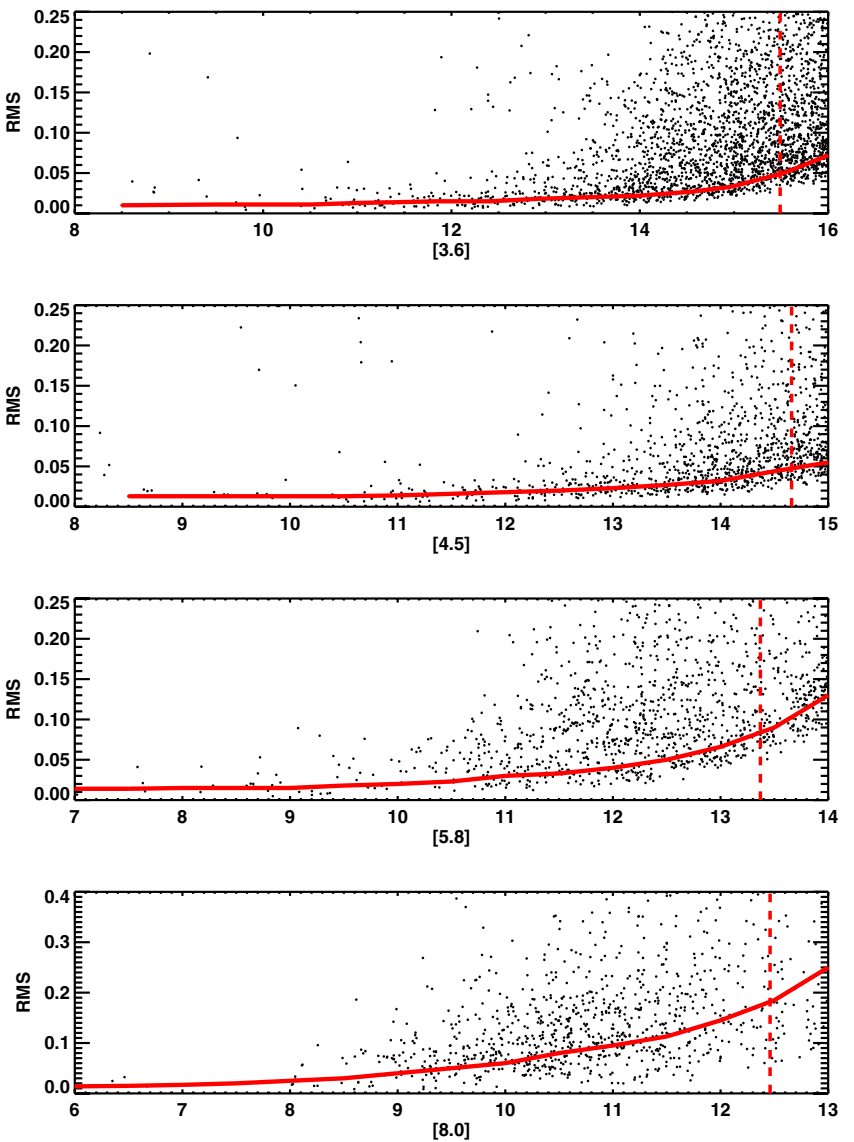

Figure 2. Light-curve rms vs. mean magnitude for all detections in the mosaic at $3.6 \mu \mathrm{m}$. The fitted polynomial (red solid line) has been used as a measure of the error. The vertical dashed line represents the magnitude of our faintest target.

(A color version of this figure is available in the online journal.)

mapping data (all channels) to analyze longer-term (up to 14 days) variability. In addition, the mapping observations will be combined to produce new deeper maps and search for new members of the globule.

\subsection{Mapping Data Photometry}

Our starting point for the data analysis was the post-Basic Calibrated Data (postBCD) mosaic image produced by the IRAC pipeline software (ver. S17.0.4). All the data have been analyzed with IRAF standard procedures. We performed aperture photometry using PHOT with a source aperture of 3 pixel radius (3".60). The aperture radius was selected in order to obtain the maximum signal-to-noise ratio $(\mathrm{S} / \mathrm{N})$. The sky background was subtracted using a 4 pixel (4".80) wide annulus. The corresponding aperture corrections, taken from the Spitzer Web site have been applied. The mean exposure time for the final mosaics is $52 \mathrm{~s}$ and we have a total of 28 exposures taken every $12 \mathrm{hr}$. Calculating appropriate photometric errors is crucial in order to apply variability tests such as the $\chi^{2}$ test and thus we decided to compute the photometric errors empirically from the data themselves. Figure 2 plots the observed photometric rms in the time series for each star detected at 3.6, 4.5, 5.8, and $8.0 \mu \mathrm{m}$ respectively, as a function of magnitude. We used a polynomial fit close to the lower envelope for each IRAC channel as the estimated photometric uncertainty for a given magnitude.

An example of the time series for one of our nonvariable targets can be seen in Figure 3. 


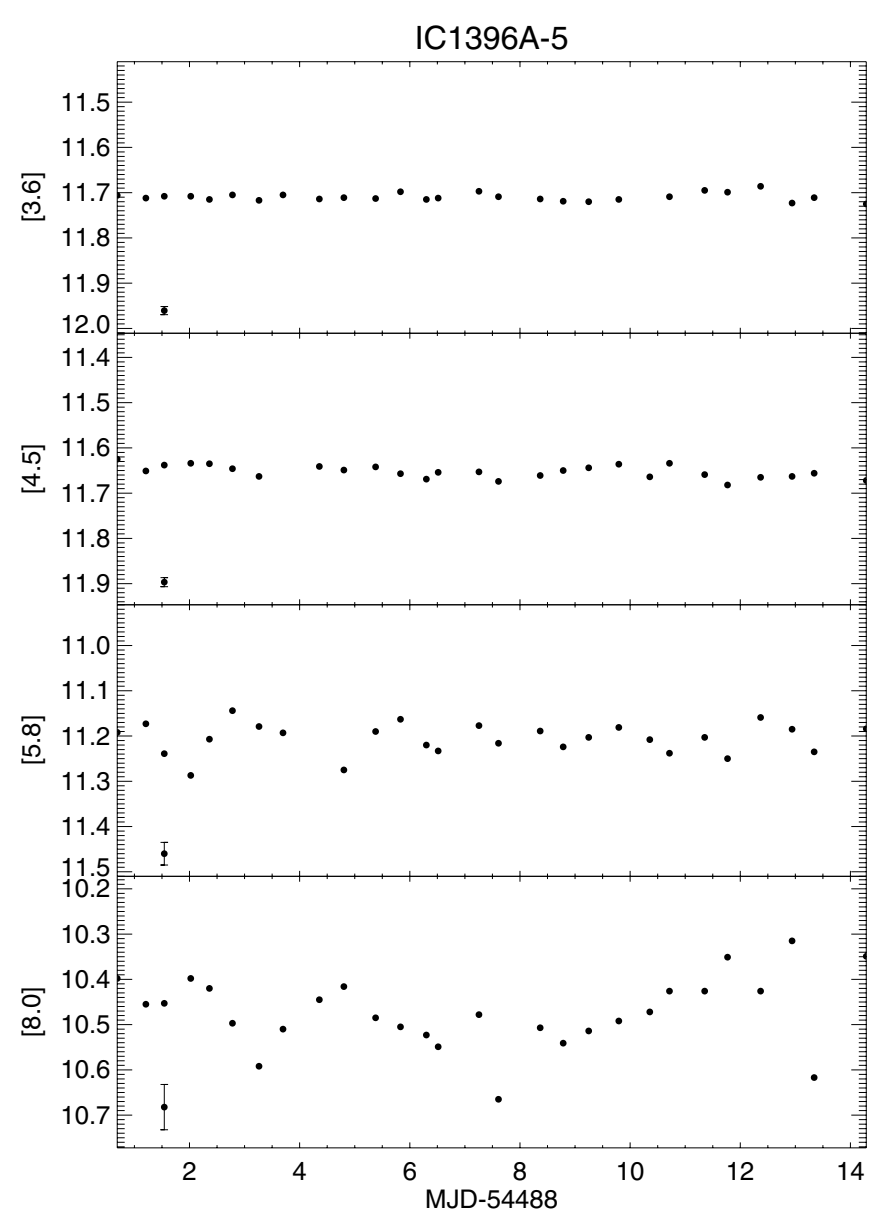

Figure 3. Example of a light curve for a nonvariable YSO, IC1396A-5, in the four IRAC bandpasses. The rms of the light curves is $9,14,34$, and $80 \mathrm{mmag}$ for IRAC channels $1-4$, respectively.

\subsection{Staring Data Photometry}

In the case of the staring data, our starting point was the individual Basic Calibrated Data (BCD) frames produced by the IRAC pipeline software (ver. S17.0.4) at the Spitzer Science Center. This pipeline produces fully flux-calibrated images, in units of $\mathrm{MJy} \mathrm{sr}^{-1}$, that have had most of the instrumental signatures removed. Some artifacts remain in the pipeline processed data, however-so we have used IDL code provided by the Spitzer Science Center to correct for column pull down and muxbleed (Ch. 1 and Ch. 2 features produced by the presence of very bright sources).

As for the mapping data, aperture photometry was performed in each image using PHOT with an aperture of 3 pixels radius, a background annulus of 4 pixels width and the appropriate aperture corrections. ${ }^{6}$ In order to improve the $\mathrm{S} / \mathrm{N}$, the $\mathrm{BCD}$ images were combined in groups of 5. Therefore, we have 400 merged data points with 1 minute increments spanning almost $7.5 \mathrm{hr}$ of observation time.

Figure 4 illustrates the pointing stability during these observations. Only a very small (less than 0.3 ) periodic movement is seen. This effect is systematic and documented in the Spitzer Web site. Such motion can affect the Ch. 2 fluxes at of order the

\footnotetext{
6 The BCD data have the native pixel scale of about $1^{\prime \prime} .22$ pixel $^{-1}$ and have not been distortion corrected, so the standard aperture correction is only approximately correct; however, since our interest is only in photometric variability, the small errors this introduces are irrelevant.
}

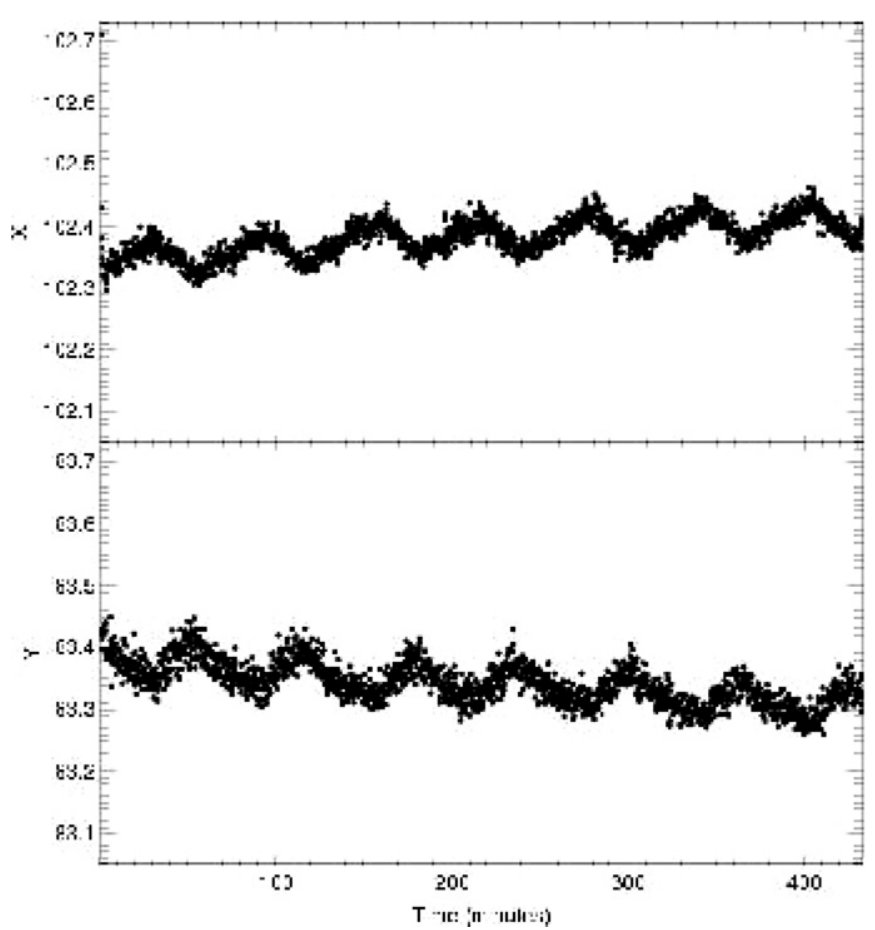

Figure 4. Array $x$-position (top) and $y$-position (bottom) as a function of time for one of our targets. Only the already known 3000 s oscillation of the pointing can be seen.

$1 \%$ level; however, because we know the pointing movements precisely, we can identify any photometric variations related to this effect. Because we see no measurable variations on the timescale of the pointing drift, we have not attempted to correct our photometry for the "pixel-phase" effects. The uncertainties in the light curves were computed empirically. We assumed that no significant real variability in our objects occurs on timescales of 10 minutes or less. We measured the scatter of every 10 data points, and the $1 \sigma$ error bars in the figures represent the median of these values.

The brightest objects of our sample show an upward trend in brightness of $<3 \%$ from the beginning to the end of the observation at Ch. 4 . Because the same trend cannot be seen in the Ch. 2 data and because this variation only appears in the brightest objects, we believe that it is due to the latent image charge buildup, an instrumental effect that was also observed in previous cases with similar kinds of data (Charbonneau et al. 2005; Morales-Calderón et al. 2006). This instrumental effect may depend on the flux of the target and there is also a pixeldependent term in the behavior of the long-term latents. In addition, it is possible that they are frametime dependent. To derive a first-order correction for this effect, we have selected a bright, nonvariable star in our data and fitted a second-order polynomial to its normalized flux. We approximately correct the fluxes for all other bright stars (approximately [8.0] $<10$ ) which show this trend, by dividing their time-series photometry by this polynomial fit.

\section{COMBINED MAPS}

In addition to the individual mosaics, we have co-added all the mapping mode observations using MOPEX in order to produce a new deeper map. The photometry has been obtained in the same way as in the previous cases and we have used the IRAC color-color diagram, [3.6]-[4.5] versus [5.8]-[8.0], to target 


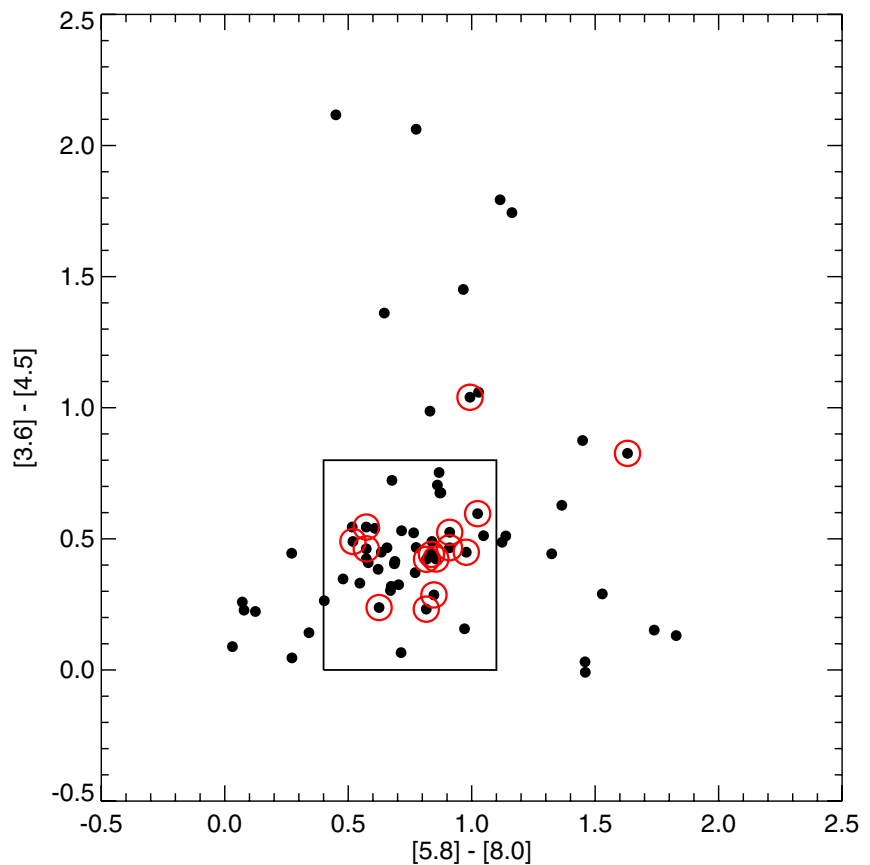

Figure 5. IRAC color-color diagram after Allen et al. (2004). Black filled circles represent the whole sample of YSOs and red open circles stand for our new Class I and Class II selections. Objects inside the box are classified as Class II objects while redder colors are indicative of Class I objects and objects close to $(0,0)$ are either field objects or Class III sources (Allen et al. 2004).

(A color version of this figure is available in the online journal.)

possible candidates and analyze their time series too. Thus, we are requiring our new candidate members to have detections at all IRAC bands. This color-color diagram was presented as a tool to separate young stars of different classes (Allen et al. 2004; Megeath et al. 2004), depending on where they fall in the diagram. Note however that Class III objects cannot be separated from field objects due to their lack of infrared excess. The Elephant Trunk Nebula is embedded in a web of bright, spatially variable nebulosity which is brightest at $8 \mu \mathrm{m}$ while normal stars are usually faint at that wavelength. Thus, we have excluded from our list of new candidates those objects showing large [5.8]-[8.0] but small [3.6]-[4.5] colors, and that are located within the brightest parts of the nebulosity or the parts with largest gradients between wavelengths. In addition, we have eliminated from our list all the objects with errors larger than $0.1 \mathrm{mag}$ in one or both of the bluer IRAC bands in order to have a reliable sample of new candidate members. Figure 5 shows the IRAC [3.6]-[4.5] versus [5.8]-[8.0] diagram with the previously known YSOs as well as our new candidates plotted.

In addition, we have used the spectral energy distribution (SED) of each source as a way to characterize the structure of its circumstellar disk. After Lada et al. (2006) we have used the 3.6$8.0 \mu \mathrm{m}$ slope for each source detected in all four IRAC bands to distinguish between objects with optically thick, primordial disks $\left(\alpha_{\text {IRAC }}>-1.8\right)$, objects surrounded by optically thin or anemic disks $\left(-2.56<\alpha_{\text {IRAC }}<-1.8\right)$ and objects without disks $\left(\alpha_{\mathrm{IRAC}}<-2.56\right)$. This is based on the distribution of the disk population in Taurus (1-2 Myr). The SEDs of our new targets can be seen in Figure 6. We have used previous observations from the Spitzer archive to derive the fluxes of our new targets at $24 \mu \mathrm{m}$ (PID:58, PI: Rieke). Most of our new candidates are located within the head of the globule, where the emission at $24 \mu \mathrm{m}$ is very intense and thus we were able to derive magnitudes for only a few of our targets. Our new candidate members with their photometry and assigned evolutionary class (by means of the IRAC color-color diagram) are provided in Table 1. All these objects have thick disks based on their IRAC SED slope.

\section{TIME SERIES AND PRESENCE OF VARIABILITY}

We have tabulated and examined the time-series photometry for all of the objects detected in the mapping and staring data. $1 \sigma$ rms uncertainties of $\sim 2$ mmag in Ch. 2 and $\sim 4$ mmag in Ch. 4 for the brightest targets are achieved for the staring data. For the mapping data the lowest uncertainties achieved are of the order of 6 mmag in the two bluest bandpasses. The light curves of all previously known and new candidate members were visually inspected. In this way different types of variability, that may be difficult to pick out in an automated fashion, can be spotted while bad photometric measurements can be eliminated. However, this process is totally subjective. Since we wanted to search for variability also in the field, and given the large number of detections, we have used the $\chi^{2}$ test and the Stetson J statistic as quantitative estimators of photometric variability for both short- and long-term variability.

The $\chi^{2}$ test determines the probability that the deviations in a light curve are consistent with the photometric errors (i.e., nonvariable). The null hypothesis for the test is that there is no variability. We evaluated the $\chi^{2}$ statistic,

$$
\chi^{2}=\sum_{k=1}^{k=K}\left(\frac{\Delta m(k)}{\sigma}\right)^{2},
$$

where $K$ is the number of data points in the light curve, $\Delta m(k)$ is the magnitude for each data point with the mean magnitude subtracted, and $\sigma$ is the rms error in the photometry. A large $\chi^{2}$ value indicates a greater deviation compared to the photometric errors and thus a smaller probability that the null hypothesis is true (i.e., variable). This probability, $p$, is calculated, and we claim evidence for variability if $p<0.01$. Because this method is very sensitive to the accuracy of the error estimation, our technique of using the data to empirically estimate the errors should minimize false detections. In addition, since a single bad measurement can produce high $\chi^{2}$ values, we eliminated from the time series the $3 \sigma$ isolated deviants prior to calculating the statistic.

The $\chi^{2}$ test does not take advantage of correlated changes in multiband time series and thus we used the Stetson variability index $J$ (Stetson 1996) to try to identify low amplitude, correlated variables. The Stetson variability index was computed for each star from the four IRAC bands magnitudes and their associated photometric uncertainties as

$$
J=\frac{\sum_{k=1}^{n} w_{k} \operatorname{sgn}\left(P_{k}\right) \sqrt{\left|P_{k}\right|}}{\sum_{k=1}^{n} w_{k}},
$$

where we assign, to each of the $n$ pairs of observations considered, a weight $w_{k}$, and

$$
P_{k}=\left\{\begin{array}{lll}
\delta_{i(k)} \delta_{j(k)} & \text { if } \quad i(k) \neq j(k) \\
\delta_{i(k)}^{2}-1 & \text { if } \quad i(k)=j(k)
\end{array}\right.
$$

is the product of the normalized residuals of the two paired observations $i$ and $j$, and

$$
\delta_{i}=\sqrt{\frac{n}{n-1}} \frac{m_{i}-\bar{m}}{\sigma_{i}}
$$


Table 1

New Candidates Photometry from the Co-added Deep Mosaics

\begin{tabular}{|c|c|c|c|c|c|c|c|c|c|c|}
\hline Object $^{\mathrm{a}}$ & R.A. (J2000), Decl. (J2000) & $J^{\mathrm{b}} \mathrm{e} J$ & $H^{\mathrm{b}} \mathrm{e} H$ & $K_{s}^{\mathrm{b}} \mathrm{e} K_{s}$ & [3.6] e[3.6] & {$[4.5] \mathrm{e}[4.5]$} & {$[5.8] \mathrm{e}[5.8]$} & {$[8.0] \mathrm{e}[8.0]$} & [24] e[24] & Class \\
\hline IC1396A-60 & $21: 36: 47.18+57: 29: 52.6$ & $14.184^{c} \ldots$ & 14.0730 .058 & 12.630 .028 & 10.4190 .003 & 9.8740 .003 & 9.4190 .008 & 8.8460 .02 & & II \\
\hline IC1396A-61 & $21: 36: 47.63+57: 29: 54.1$ & $13.568^{\mathrm{c}} \ldots$ & 12.3420 .042 & 11.6550 .033 & 10.4260 .004 & 9.9640 .004 & 9.5450 .01 & 8.9720 .031 & & II \\
\hline IC1396A-62 & $21: 37: 14.51+57: 28: 40.9$ & 14.5110 .039 & 13.5590 .041 & 12.9540 .034 & 11.8390 .002 & 11.3490 .002 & 11.1280 .002 & 10.6090 .01 & 7.0120 .039 & II \\
\hline IC1396A-63 & $21: 37: 17.42+57: 29: 27.5$ & 14.1220 .043 & 13.1350 .043 & 12.5830 .037 & 11.8590 .003 & 11.4360 .004 & 11.1040 .003 & 10.2490 .008 & 7.1650 .054 & II \\
\hline IC1396A-64 ${ }^{\mathrm{d}}$ & $21: 37: 17.37+57: 29: 20.8$ & $14.091^{\mathrm{c}} \ldots$ & 13.5570 .071 & $13.13^{\mathrm{c}} \ldots$ & 12.9010 .012 & 12.6690 .013 & 12.3440 .011 & 11.5280 .023 & & II \\
\hline IC1396A-65 & $21: 36: 42.49+57: 25: 23.3$ & 14.8060 .043 & 14.0890 .047 & 13.7580 .049 & 13.1940 .007 & 12.9560 .007 & 12.5530 .01 & 11.9280 .041 & 8.9590 .158 & II \\
\hline IC1396A-66 & $21: 37: 05.87+57: 32: 12.5$ & 15.130 .051 & 14.2840 .059 & 14.1390 .078 & 13.3720 .012 & 13.0860 .012 & 12.80 .066 & 11.9530 .123 & $\ldots$ & II \\
\hline IC1396A-67 & $21: 35: 58.52+57: 29: 15.1$ & 16.5820 .167 & 15.3730 .125 & 14.6320 .1 & 13.5370 .019 & 13.0880 .013 & 12.5340 .04 & 11.5560 .121 & & II \\
\hline IC1396A-68 & $21: 36: 56.26+57: 29: 52.3$ & $18.527^{\mathrm{c}} \ldots$ & $16.098^{c} \ldots$ & 15.20 .141 & 13.5770 .03 & 12.9810 .022 & 12.150 .096 & 11.1260 .224 & & II \\
\hline IC1396A-69 & $21: 36: 38.03+57: 26: 57.9$ & 15.6040 .072 & 14.8930 .073 & 14.5230 .08 & 13.580 .009 & 13.1580 .007 & 12.7960 .011 & 11.9790 .02 & 8.1790 .137 & II \\
\hline IC1396A-70 & $21: 36: 12.60+57: 31: 26.3$ & 16.5040 .132 & $16.161^{\mathrm{c}} \ldots$ & 15.1360 .14 & 14.0060 .019 & 13.4810 .012 & 13.340 .077 & 12.4290 .195 & & II \\
\hline IC1396A-71 & $21: 36: 40.34+57: 25: 45.7$ & 16.2710 .094 & 15.2030 .099 & 14.8670 .113 & 14.0590 .009 & 13.6180 .008 & 13.2030 .016 & 12.3660 .022 & 9.0510 .149 & II \\
\hline IC1396A-72 & $21: 36: 18.97+57: 29: 05.1$ & & & & 14.1970 .014 & 13.1570 .007 & 12.290 .022 & 11.2970 .059 & & I \\
\hline IC1396A-73 & $21: 37: 11.78+57: 30: 34.9$ & 16.1750 .115 & $15.52^{\mathrm{c}} \ldots$ & 14.9280 .148 & 14.2890 .013 & 13.8230 .015 & 13.3720 .036 & 12.4620 .057 & 6.3050 .072 & II \\
\hline IC1396A-74 & $21: 36: 36.35+57: 32: 09.3$ & $\ldots$ & $\ldots$ & $\ldots$ & 15.5050 .085 & 14.6790 .02 & 13.2370 .104 & 11.6060 .101 & $\ldots$ & I \\
\hline
\end{tabular}

Notes.

${ }^{a}$ Sicilia-Aguilar et al. (2006) identified 57 YSOs in this region and there are another two objects that were identified by Reach et al. (2005), thus we name our new candidate members beginning with number 60.

${ }^{\mathrm{b}}$ Photometry for the $J, H$, and $K_{s}$ bandpasses has been taken from the 2MASS database (Cutri et al. 2003).

${ }^{\mathrm{c}}$ Upper limits.

d This object is very faint at $24 \mu \mathrm{m}$ and it is located too close to a brighter object to derive a reliable magnitude. 

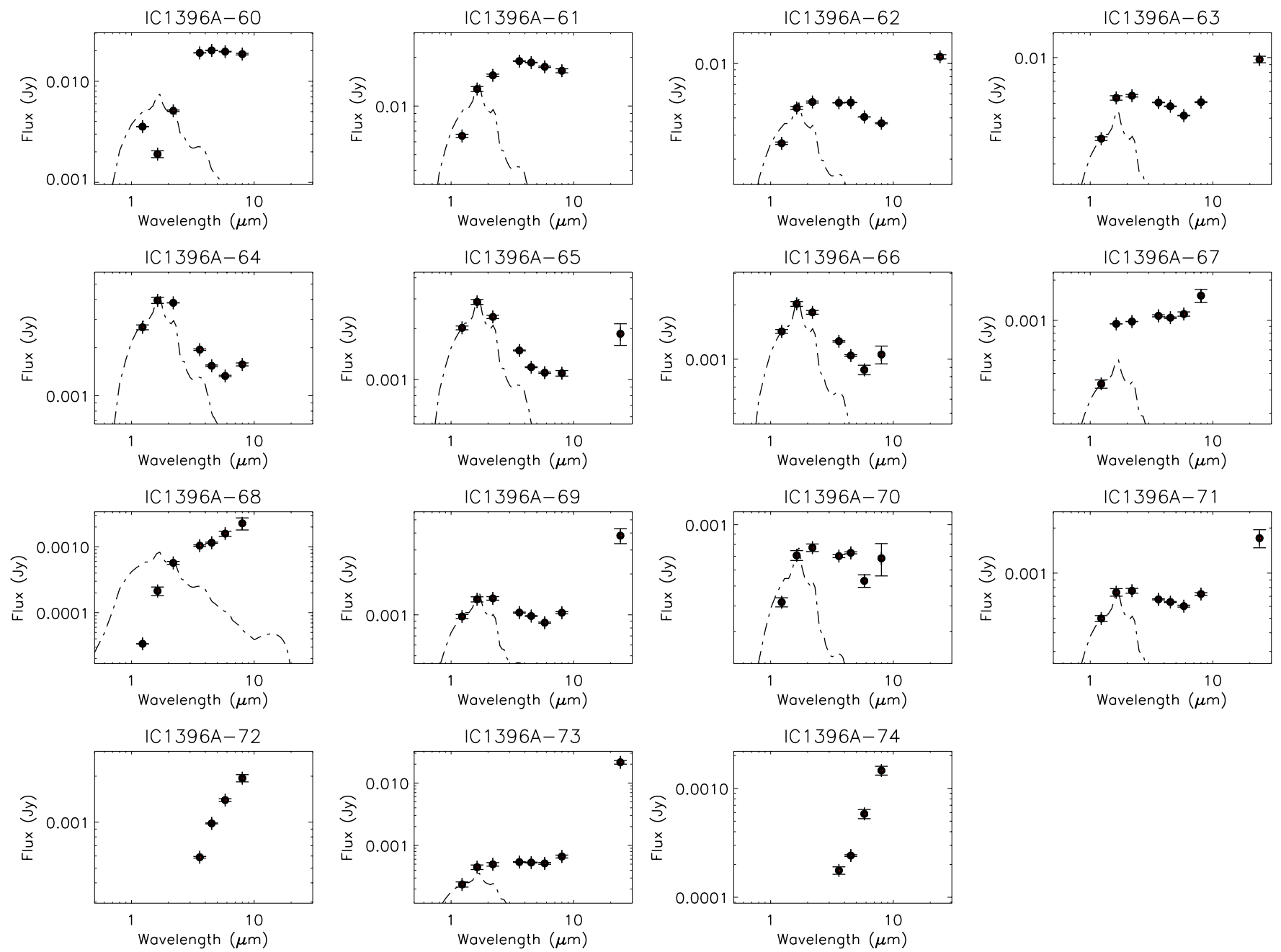

Figure 6. SEDs for our new candidates. All of them have been classified as Class II or Class I objects by means of the IRAC color-color diagram and have thick disks surrounding the central object. The $J, H$, and $K_{s}$ magnitudes come from the 2MASS database (Cutri et al. 2003). The dashed lines are Kurucz models (Castelli et al. 1997) aiming to represent the photosphere of each target assuming that the bluest 2MASS data point with good quality photometry represents the photospheric flux.

is the magnitude residual of a given observation from the average normalized by the standard error. For a nonvariable star, with only random noise, the Stetson variability index should be scattered around zero and have higher, positive values for stars with correlated physical variability. Figure 7 shows the Stetson statistic as a function of the [3.6] magnitude. The dashed line at $J=0$ shows the expected value of the variability index for non variable stars, and the dotted line at $J=0.55$ represents the minimum value adopted by Carpenter et al. (2001) in a similar near-IR variability study toward a several square degree area in the Orion. Most of our targets have magnitudes brighter than [3.6] $=13.5$ and only one (cataloged by both the $\chi^{2}$ test and the $J$ statistic as nonvariable) has a magnitude fainter than $[3.6]=14.2$. Following the choice adopted by Carpenter et al. (2001), and based on our own comparison of the $\chi^{2}$ and $J$ statistic results for individual stars, we adopt $J=0.55$ as the borderline between variable and nonvariable objects. However, we note that the $J$ index shows higher dispersion toward fainter magnitudes and a different minimum value should be used for fainter objects.

If evidence of variability was found in an object, we looked for a periodic signal in the data following the methodology described by Scargle (1982). This method is equivalent to a least-squares fit (in the time domain) of sinusoids to the data.

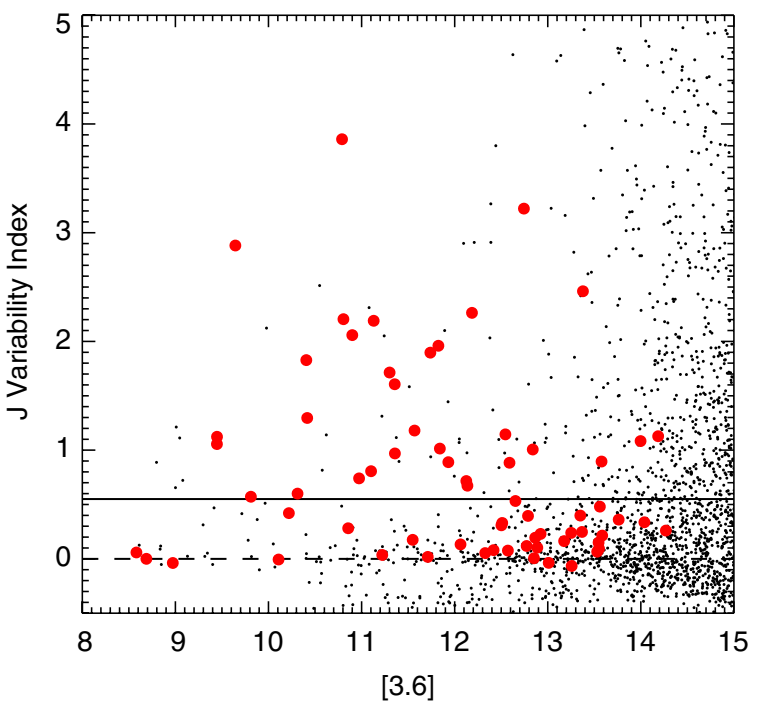

Figure 7. Stetson variability index $(J)$ plotted as a function of [3.6] mean magnitude for stars brighter than $[3.6]=15$. The dashed line at $J=0$ shows the expected value of the variability index for nonvariable stars, and the dotted line at $J=0.55$ represents the minimum adopted value used to identify variable stars in this study. Our IC1396A YSOs are represented with red filled circles.

(A color version of this figure is available in the online journal.) 
The algorithm calculates the normalized Lomb periodogram for the data and gives us a false-alarm probability based on the peak height in the periodogram as a measure of significance. There is, in general a good agreement between the periods found at different wavelengths, however caution should be taken since in most cases our data only cover a time frame of the order of the estimated period.

\section{RESULTS AND DISCUSSION}

\subsection{The Mapping Data: Long-term Variable Members}

Fifty two previously known YSOs from Sicilia-Aguilar et al. (2006) and 13 YSOs from Reach et al. (2004; 11 of them are in common with the sample from Sicilia-Aguilar et al. 2006) plus 15 new candidates fall inside the area of our mosaics, and most of them have good photometry in the four IRAC bands. As the first step to determine their variability, we applied the $\chi^{2}$ test to the Ch. 1 and Ch. 2 data. We did not calculate $\chi^{2}$ for the longer wavelength channels because those data are much noisier. Forty stars were labeled by the $\chi^{2}$ statistics as variable objects in IRAC Ch. 1 and Ch. 2 while four objects, IC1396A62, IC1396A-63, IC1396A-66, and IC1396A-72, were found to be variable only in Ch. 1. Two of these objects, IC1396A-62 and IC1396A-63 are located near the border of the Ch. 2 and Ch. 4 mosaics and are outside the FOV in most of them; however, the light curve built for these objects with the photometry of Ch. 3 confirms the variation shown in Ch. 1. IC1396A-66 shows low-amplitude variations in $\mathrm{Ch}$. 1 but the light curves at longer wavelengths, even when they follow the same trend, are too noisy to confirm the variation. Object 72 has been detected in the deep mosaics which have been built combining all the individual maps, however, in the individual observations only the $\mathrm{Ch} .1$ data are good enough to build its time series. We thus cannot confirm the Ch. 1 result, and consider object 72 nonvariable.

As the other primary means to determine variability, we also calculated the Stetson $J$ variability index using the four IRAC bands for each source. Twelve objects labeled as variable by the $\chi^{2}$ test were labeled as nonvariable by the $J$ index however, all but three of them (objects 19, 30, and 51) are labeled as variable if one excludes Ch. 4 to calculate the $J$ statistic. We have given a final tag-variable $(V)$ or nonvariable $(N)$ - to each source based on the $\chi^{2}$ test, the $J$ index, and a careful visual inspection of all the light curves. The very good similarity, in most cases, between the light-curve shape for $\mathrm{Ch} .1$ and for Ch. 2 (and 3 and 4 when the $\mathrm{S} / \mathrm{N}$ is good enough) is good evidence that the variability is real. Only one star showed what we believe is real variability in Ch. 3 or 4 but not in the shorter wavelength channels. That object is IC1396A-74 (see later discussion and Figure 11(b)).

Table 2 presents the results of all targets including IRAC magnitudes, rms amplitudes, result of the $\chi^{2}$ test, $J$ statistic, our final variability tag, and periods for $\mathrm{Ch} .1$ and $\mathrm{Ch}$. 2. Note the use of the term $T_{\mathrm{var}}$ instead of rotation period in the table indicating that these are variability timescales rather than periods since the timespan of our observations are not long enough to confirm the periodicity. Objects are named following the order in Table 6 of Sicilia-Aguilar et al. (2006) until number 57. Objects 58 and 59 are YSOs from Reach et al. (2004) not in common with SiciliaAguilar et al. (2006), and objects from 60 to 74 are our 15 new candidates. YSO classifications for each object derived from the IRAC color-color diagram are also included as well as the YSO classification previously given for these objects. There are eight objects marked as having different classification from that in Sicilia-Aguilar et al. (2006). Four of these as Class I by one of us, and Class II by the other, but are really very close to the boundary between those classes. Actually two of those objects (1 and 40) are in common with Reach et al. (2004) where they were classified as Class I/II. The remaining four objects were classified by us as Class III because of the lack of IR excess. One of them ( -30 , previously known as LKHa 349a) was also studied by Reach et al. (2004) and classified as Class III. The remaining three objects could be field stars (two of them, 9 and 32 were classified by Sicilia-Aguilar et al. (2006) as possible nonmembers).

There is a range of shapes found in the light curves and Figures 8-12 present the different observed mid-infrared variability characteristics. All variable stars have their photometry tabulated in Table 3, available in the online version of this article. About $30 \%$ of our YSOs show light curves that resemble the optical variations of BY Dra variables (spotted stars whose apparent luminosities vary due to rotational modulation-Byrne et al. 1987). T Tauri stars also show such variability in the optical and near-IR, generally attributed to either cool, solar-type spots or hot spots associated with accretion flows onto the stellar photosphere (Rydgren \& Vrba 1983; Bouvier et al. 1986; Vrba et al. 1986). It is not obvious, however, whether our mid-IR light curves can be attributed to such spots (see discussion in Section 6). Figure 8 shows these periodic-like light curves, where the characteristic timescale of variability increases from the upper left panel to the lower right one. The peak-to-peak variability amplitudes of these objects can be found in Table 4. These data illustrate that the variability amplitudes are essentially invariant with wavelength from 3.6 to $8 \mu \mathrm{m}$.

Other types of variability exhibited by one or more of our YSOs include the following.

1. Rapid (timescales of hours) variability inconsistent with rotational modulation. No apparent color dependence during the variations. Only two objects present this kind of variations: IC1396A-39 (object $\eta$ in Reach et al. 2004) and IC1396A-62, see Figure 9. Objects 39 and 62 are Class I and Class II, respectively. Possible causes: flares or accretion flickering.

2. Slow (timescales of days), nonperiodic variability changes, with little or no color changes. $20 \%$ of our sample of YSOs fall under this category. Their light curves are shown in Figure 10. Only three Class I YSOs (IC1396A-1, IC1396A8, and IC1396A-50) are included in this group. Possible causes: accretion variations; rapidly evolving spots. We admit there is in some cases ambiguity whether an object should be in Figure 8 or 10 - the timespan of our observations is too short for certainty.

3. Slow (timescales of days), flux modulation with color dependence. Three objects of our sample show this kind of variations: IC1396A-57 (Figure 11(a)), which shows a slow brightening over the entire 14 days of observation with a slower rise to maximum going to longer wavelengths, and IC1396A-35 (Object $\beta$ in Reach et al. 2004; Figure 12), which shows a slow fading over about 5 days, followed by a sudden reset to the original brightness and then approximately constant flux at 3.6 and $4.5 \mu \mathrm{m}$ for the remaining time. IC1396A-35 is the only YSO with significant, coherent color variation over the 14 day observing runbecoming significantly redder when faint. Another possible exemplar of this class is IC1396A-74, which shows essentially constant magnitude at 3.6, 4.5, and $5.8 \mu \mathrm{m}$, but 
Table 2

Long-term Variability: Main Results from Mapping Data

\begin{tabular}{|c|c|c|c|c|c|c|c|c|c|c|c|c|c|c|}
\hline \multirow[t]{2}{*}{ Object $^{\mathrm{a}}$} & \multirow{2}{*}{$\begin{array}{c}\text { R.A. Decl. } \\
\text { hr, deg (J2000) }\end{array}$} & \multirow{2}{*}{$\begin{array}{l}\text { Class }^{\mathrm{b}} \\
\text { SA06 }\end{array}$} & \multirow{2}{*}{$\begin{array}{c}\text { ID, } \text { Class }^{\mathrm{c}} \\
\text { R04 }\end{array}$} & \multirow[t]{2}{*}{ Class $^{\mathrm{d}}$} & \multicolumn{4}{|c|}{$3.6 \mu \mathrm{m}$} & \multicolumn{4}{|c|}{$4.5 \mu \mathrm{m}$} & \multirow[t]{2}{*}{$J$ index } & \multirow{2}{*}{$\begin{array}{c}\text { Var } \\
\text { Final } \\
\end{array}$} \\
\hline & & & & & mag & $\mathrm{rms}$ & $\chi^{2}$ & $T_{\mathrm{var}}$ (days) & mag & $\mathrm{rms}$ & $\chi^{2}$ & $T_{\text {var }}$ (days) & & \\
\hline IC1396A-1 & $21: 35: 57.93+57: 29: 09.9$ & II & $\xi, \mathrm{I} / \mathrm{II}$ & $\mathrm{I}^{\mathrm{e}}$ & $13.378 \pm 0.015$ & 0.101 & $\mathrm{~V}$ & $\ldots$ & $12.752 \pm 0.017$ & 0.106 & $\mathrm{~V}$ & $\ldots$ & 2.46 & $\mathrm{~V}$ \\
\hline IC1396A-2 & $21: 35: 59.05+57: 30: 23.3$ & II & & II & $12.516 \pm 0.012$ & 0.023 & $\mathrm{~V}$ & $\ldots$ & $12.200 \pm 0.013$ & 0.026 & $\mathrm{~V}$ & $\ldots$ & 0.33 & $\mathrm{~V}$ \\
\hline IC1396A-3 & $21: 36: 06.06+57: 26: 34.1$ & II & & $\mathrm{I}^{\mathrm{e}}$ & $13.010 \pm 0.015$ & 0.02 & $\mathrm{~N}$ & $\ldots$ & $12.567 \pm 0.016$ & 0.024 & $\mathrm{~N}$ & $\ldots$ & -0.04 & $\mathrm{~N}$ \\
\hline IC1396A-4 & $21: 36: 07.98+57: 26: 37.1$ & I & $\gamma, \mathrm{I} / 0$ & I & $10.805 \pm 0.006$ & 0.061 & $\mathrm{~V}$ & $\ldots$ & $9.026 \pm 0.009$ & 0.048 & $\mathrm{~V}$ & $\ldots$ & 2.20 & $\mathrm{~V}$ \\
\hline IC1396A-5 & $21: 36: 03.89+57: 27: 12.1$ & II & & II & $11.710 \pm 0.009$ & 0.009 & $\mathrm{~N}$ & $\ldots$ & $11.652 \pm 0.009$ & 0.014 & $\mathrm{~N}$ & $\ldots$ & 0.02 & $\mathrm{~N}$ \\
\hline IC1396A-6 & $21: 36: 07.46+57: 26: 43.6$ & I & & I & $12.836 \pm 0.013$ & 0.032 & $\mathrm{~V}$ & 4.9 & $11.027 \pm 0.009$ & 0.022 & $\mathrm{~V}$ & 5.1 & 1.01 & $\mathrm{~V}$ \\
\hline IC1396A-7 & $21: 36: 18.36+57: 28: 31.6$ & I & $\epsilon, \mathrm{I} / 0$ & I & $12.744 \pm 0.012$ & 0.079 & $\mathrm{~V}$ & $\ldots$ & $10.701 \pm 0.009$ & 0.083 & $\mathrm{~V}$ & $\ldots$ & 3.22 & $\mathrm{~V}$ \\
\hline IC1396A-8 & $21: 36: 19.42+57: 28: 38.5$ & I & $\delta, \mathrm{I} / 0$ & I & $12.792 \pm 0.012$ & 0.033 & $\mathrm{~V}$ & $\ldots$ & $11.050 \pm 0.009$ & 0.023 & $\mathrm{~V}$ & $\ldots$ & 0.39 & $\mathrm{~V}$ \\
\hline IC1396A-9f & $21: 36: 14.20+57: 27: 37.9$ & II & & $\mathrm{III}^{\mathrm{e}}$ & $8.972 \pm 0.006$ & 0.027 & $\mathrm{~N}$ & $\ldots$ & $8.893 \pm 0.009$ & 0.009 & $\mathrm{~N}$ & $\ldots$ & -0.03 & $\mathrm{~N}$ \\
\hline IC1396A-10 f & $21: 36: 14.20+57: 27: 57.7$ & II & & II & $13.531 \pm 0.016$ & 0.034 & $\mathrm{~N}$ & $\ldots$ & $13.610 \pm 0.028$ & 0.036 & $\mathrm{~N}$ & $\ldots$ & 0.07 & $\mathrm{~N}$ \\
\hline IC1396A-11 & $21: 36: 16.64+57: 28: 40.4$ & I & & I & $12.502 \pm 0.012$ & 0.025 & $\mathrm{~V}$ & $\ldots$ & $11.751 \pm 0.009$ & 0.022 & $\mathrm{~V}$ & $\ldots$ & 0.31 & $\mathrm{~V}$ \\
\hline IC1396A-12 & $21: 36: 16.99+57: 26: 39.9$ & II & & II & $12.852 \pm 0.012$ & 0.02 & $\mathrm{~N}$ & $\ldots$ & $12.501 \pm 0.015$ & 0.012 & $\mathrm{~N}$ & $\ldots$ & 0.01 & $\mathrm{~N}$ \\
\hline IC1396A-13 & $21: 36: 23.68+57: 32: 45.2$ & II & & II & $12.589 \pm 0.012$ & 0.075 & $\mathrm{~V}$ & 9.1 & $12.374 \pm 0.014$ & 0.071 & $\mathrm{~V}$ & 9.1 & 0.88 & $\mathrm{~V}$ \\
\hline IC1396A-14 & $21: 36: 25.07+57: 27: 50.2$ & II & & II & $12.545 \pm 0.012$ & 0.039 & $\mathrm{~V}$ & $\ldots$ & $12.041 \pm 0.012$ & 0.05 & $\mathrm{~V}$ & $\ldots$ & 1.14 & $\mathrm{~V}$ \\
\hline IC1396A-15 & $21: 36: 33.00+57: 28: 49.3$ & II & & II & $13.765 \pm 0.018$ & 0.029 & $\mathrm{~V}$ & $\ldots$ & $13.355 \pm 0.024$ & 0.044 & V & $\ldots$ & 0.36 & $\mathrm{~V}$ \\
\hline IC1396A-16 & $21: 36: 35.31+57: 29: 31.1$ & II & & II & $11.356 \pm 0.006$ & 0.038 & $\mathrm{~V}$ & $\ldots$ & $10.993 \pm 0.009$ & 0.042 & $\mathrm{~V}$ & $\ldots$ & 1.61 & $\mathrm{~V}$ \\
\hline IC1396A-17 & $21: 36: 36.91+57: 31: 32.6$ & II & $\iota, \mathrm{II}$ & II & $11.102 \pm 0.006$ & 0.033 & $\mathrm{~V}$ & $\ldots$ & $10.625 \pm 0.009$ & 0.035 & $\mathrm{~V}$ & $\ldots$ & 0.81 & $\mathrm{~V}$ \\
\hline IC1396A-18 & $21: 36: 38.41+57: 29: 17.4$ & II & & II & $11.353 \pm 0.006$ & 0.049 & $\mathrm{~V}$ & $\ldots$ & $10.975 \pm 0.009$ & 0.039 & $\mathrm{~V}$ & $\ldots$ & 0.97 & $\mathrm{~V}$ \\
\hline IC1396A-19 & $21: 36: 39.15+57: 29: 53.3$ & II & $\theta, \mathrm{II}$ & II & $8.581 \pm 0.006$ & 0.071 & $\mathrm{~V}$ & $\ldots$ & $7.859 \pm 0.009$ & 0.064 & $\mathrm{~V}$ & $\ldots$ & 0.06 & $\mathrm{~N}$ \\
\hline IC1396A-20 & $21: 36: 41.46+57: 30: 27.8$ & II & & II & $12.773 \pm 0.012$ & 0.017 & $\mathrm{~N}$ & $\ldots$ & $12.383 \pm 0.014$ & 0.014 & $\mathrm{~N}$ & $\ldots$ & 0.12 & $\mathrm{~N}$ \\
\hline IC1396A-21 & $21: 36: 41.65+57: 32: 17.5$ & II & & $\mathrm{I}^{\mathrm{e}}$ & $12.653 \pm 0.012$ & 0.03 & $\mathrm{~V}$ & $\ldots$ & $12.165 \pm 0.012$ & 0.032 & $\mathrm{~V}$ & $\ldots$ & 0.53 & $\mathrm{~V}$ \\
\hline IC1396A-22 & $21: 36: 43.98+57: 29: 28.7$ & II & & II & $11.301 \pm 0.006$ & 0.069 & $\mathrm{~V}$ & 8.2 & $10.761 \pm 0.009$ & 0.047 & $\mathrm{~V}$ & 9 & 1.71 & $\mathrm{~V}$ \\
\hline IC1396A-23 ${ }^{\mathrm{f}}$ & $21: 36: 44.00+57: 28: 46.8$ & II & & II & $12.416 \pm 0.009$ & 0.016 & $\mathrm{~N}$ & $\ldots$ & $12.420 \pm 0.014$ & 0.015 & $\mathrm{~N}$ & $\ldots$ & 0.08 & $\mathrm{~N}$ \\
\hline IC1396A-24 & $21: 36: 45.96+57: 29: 33.9$ & I & & I & $9.644 \pm 0.006$ & 0.056 & $\mathrm{~V}$ & 6 & $9.114 \pm 0.009$ & 0.064 & $\mathrm{~V}$ & 6 & 2.88 & $\mathrm{~V}$ \\
\hline IC1396A-25 & $21: 36: 46.60+57: 29: 38.4$ & I & $\alpha, \mathrm{I}$ & I & $10.217 \pm 0.006$ & 0.011 & $\mathrm{~N}$ & $\ldots$ & $8.776 \pm 0.009$ & 0.012 & $\mathrm{~N}$ & $\ldots$ & 0.42 & $\mathrm{~N}$ \\
\hline IC1396A-28f & $21: 36: 47.88+57: 31: 30.6$ & I & & I & $12.137 \pm 0.009$ & 0.059 & $\mathrm{~V}$ & 5.4 & $11.862 \pm 0.009$ & 0.043 & $\mathrm{~V}$ & $\ldots$ & 0.67 & $\mathrm{~V}$ \\
\hline IC1396A-29 & $21: 36: 49.41+57: 31: 22.0$ & II & & II & $9.446 \pm 0.006$ & 0.04 & $\mathrm{~V}$ & 7.8 & $9.031 \pm 0.009$ & 0.034 & $\mathrm{~V}$ & $\ldots$ & 1.06 & $\mathrm{~V}$ \\
\hline IC1396A-30 & $21: 36: 50.72+57: 31: 10.6$ & II & LkHa349a,III & $\mathrm{III}^{\mathrm{e}}$ & $8.688 \pm 0.006$ & 0.033 & $\mathrm{~V}$ & $\ldots$ & $8.439 \pm 0.009$ & 0.035 & $\mathrm{~V}$ & $\ldots$ & $2.3 E-4$ & $\mathrm{~N}$ \\
\hline IC1396A-31 & $21: 36: 52.81+57: 29: 43.8$ & II & & II & $12.572 \pm 0.012$ & 0.011 & $\mathrm{~N}$ & $\ldots$ & $12.269 \pm 0.013$ & 0.011 & $\mathrm{~N}$ & $\ldots$ & 0.08 & $\mathrm{~N}$ \\
\hline IC1396A-32 & $21: 36: 54.50+57: 30: 05.1$ & II & & $\mathrm{III}^{\mathrm{e}}$ & $10.108 \pm 0.006$ & 0.019 & $\mathrm{~N}$ & $\ldots$ & $9.889 \pm 0.009$ & 0.006 & $\mathrm{~N}$ & $\ldots$ & -0.01 & $\mathrm{~N}$ \\
\hline IC1396A-33 ${ }^{\mathrm{f}}$ & $21: 36: 54.75+57: 31: 45.0$ & II & & II & $13.354 \pm 0.015$ & 0.03 & $\mathrm{~N}$ & $\ldots$ & 然 & 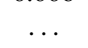 & $\ldots$ & $\ldots$ & 0.40 & $\mathrm{~N}$ \\
\hline IC1396A-34 & $21: 36: 54.89+57: 30: 00.3$ & I & $\lambda, \mathrm{I}$ & I & $11.932 \pm 0.009$ & 0.042 & $\mathrm{~V}$ & $\ldots$ & $11.251 \pm 0.009$ & 0.037 & $\mathrm{~V}$ & $\ldots$ & 0.89 & $\mathrm{~V}$ \\
\hline IC1396A-35 & $21: 36: 55.20+57: 30: 30.1$ & II & $\beta, \mathrm{II}$ & II & $9.811 \pm 0.006$ & 0.053 & $\mathrm{~V}$ & $\ldots$ & $9.309 \pm 0.009$ & 0.033 & $\mathrm{~V}$ & $\ldots$ & 0.57 & $\mathrm{~V}$ \\
\hline IC1396A-36 & $21: 36: 55.43+57: 31: 39.1$ & II & & $\mathrm{III}^{\mathrm{e}}$ & $11.222 \pm 0.006$ & 0.009 & $\mathrm{~N}$ & $\ldots$ & $11.187 \pm 0.009$ & 0.008 & $\mathrm{~N}$ & $\ldots$ & 0.04 & $\mathrm{~N}$ \\
\hline IC1396A-37 & $21: 36: 56.98+57: 29: 22.7$ & II & & II & $11.569 \pm 0.009$ & 0.049 & $\mathrm{~V}$ & 9.1 & $11.049 \pm 0.009$ & 0.041 & $\mathrm{~V}$ & 9.1 & 1.18 & $\mathrm{~V}$ \\
\hline IC1396A-38 & $21: 36: 57.67+57: 27: 33.1$ & II & & II & $9.447 \pm 0.006$ & 0.033 & $\mathrm{~V}$ & $\ldots$ & $8.987 \pm 0.009$ & 0.049 & $\mathrm{~V}$ & $\ldots$ & 1.12 & $\mathrm{~V}$ \\
\hline IC1396A-39 & $21: 36: 57.83+57: 30: 56.1$ & I & $\eta, \mathrm{I}$ & I & $12.186 \pm 0.009$ & 0.057 & $\mathrm{~V}$ & 7.8 & $11.139 \pm 0.009$ & 0.058 & $\mathrm{~V}$ & 9 & 2.26 & $\mathrm{~V}$ \\
\hline IC1396A-40 & $21: 36: 57.93+57: 29: 10.7$ & I & $v, \mathrm{I} / \mathrm{II}$ & $\mathrm{II}^{\mathrm{e}}$ & $10.316 \pm 0.006$ & 0.025 & $\mathrm{~V}$ & 6 & $9.916 \pm 0.009$ & 0.027 & $\mathrm{~V}$ & 7.6 & 0.60 & $\mathrm{~V}$ \\
\hline IC1396A-41 & $21: 36: 58.90+57: 30: 29.2$ & I & & I & $12.865 \pm 0.012$ & 0.032 & V & $\ldots$ & $12.211 \pm 0.013$ & 0.027 & V & $\ldots$ & 0.19 & $\mathrm{~V}$ \\
\hline
\end{tabular}


Table 2

\begin{tabular}{|c|c|c|c|c|c|c|c|c|c|c|c|c|c|c|}
\hline \multirow[t]{2}{*}{ Object $^{\mathrm{a}}$} & \multirow{2}{*}{$\begin{array}{c}\text { R.A. Decl. } \\
\text { hr, deg (J2000) }\end{array}$} & \multirow{2}{*}{$\begin{array}{l}\text { Class }^{b} \\
\text { SA06 }\end{array}$} & \multirow{2}{*}{$\begin{array}{c}\text { ID, Class }{ }^{\mathrm{c}} \\
\text { R04 }\end{array}$} & \multirow[t]{2}{*}{ Class $^{\mathrm{d}}$} & \multicolumn{4}{|c|}{$3.6 \mu \mathrm{m}$} & \multicolumn{4}{|c|}{$4.5 \mu \mathrm{m}$} & \multirow[t]{2}{*}{$J$ index } & \multirow{2}{*}{$\begin{array}{c}\text { Var } \\
\text { Final } \\
\end{array}$} \\
\hline & & & & & mag & $\mathrm{rms}$ & $\chi^{2}$ & $T_{\text {var }}$ (days) & mag & $\mathrm{rms}$ & $\chi^{2}$ & $T_{\text {var }}$ (days) & & \\
\hline IC1396A-43 & $21: 36: 59.47+57: 31: 34.9$ & II & & II & $11.739 \pm 0.009$ & 0.055 & $\mathrm{~V}$ & 10.5 & $11.251 \pm 0.009$ & 0.063 & $\mathrm{~V}$ & $\ldots$ & 1.90 & $\mathrm{~V}$ \\
\hline IC1396A-44 ${ }^{\mathrm{f}}$ & $21: 37: 01.05+57: 30: 39.7$ & I & & I & $13.257 \pm 0.015$ & 0.023 & $\mathrm{~N}$ & $\ldots$ & $13.110 \pm 0.021$ & 0.026 & $\mathrm{~N}$ & $\ldots$ & -0.06 & $\mathrm{~N}$ \\
\hline IC1396A-45 & $21: 37: 01.91+57: 28: 22.2$ & II & & II & $10.856 \pm 0.006$ & 0.027 & $\mathrm{~V}$ & 6 & $10.535 \pm 0.009$ & 0.034 & $\mathrm{~V}$ & 6 & 0.28 & V \\
\hline IC1396A-46 & $21: 37: 02.00+57: 31: 55.3$ & II & & II & $12.061 \pm 0.009$ & 0.017 & $\mathrm{~N}$ & $\ldots$ & $11.911 \pm 0.009$ & 0.015 & $\mathrm{~N}$ & $\ldots$ & 0.13 & $\mathrm{~N}$ \\
\hline IC1396A-47 & $21: 37: 02.32+57: 31: 15.2$ & I & $\zeta, \mathrm{I}$ & I & $10.790 \pm 0.006$ & 0.065 & $\mathrm{~V}$ & 9 & $9.807 \pm 0.009$ & 0.078 & V & 9 & 3.86 & $\mathrm{~V}$ \\
\hline IC1396A-49 & $21: 37: 06.49+57: 32: 31.6$ & II & & II & $10.900 \pm 0.006$ & 0.064 & $\mathrm{~V}$ & $\ldots$ & $10.459 \pm 0.009$ & 0.068 & $\mathrm{~V}$ & $\ldots$ & 2.06 & $\mathrm{~V}$ \\
\hline IC1396A-50 & $21: 37: 07.18+57: 31: 27.8$ & I & & I & $13.585 \pm 0.016$ & 0.04 & $\mathrm{~V}$ & $\ldots$ & $12.718 \pm 0.017$ & 0.027 & $\mathrm{~V}$ & $\ldots$ & 0.21 & $\mathrm{~V}$ \\
\hline IC1396A-51 ${ }^{\mathrm{f}}$ & $21: 37: 07.71+57: 32: 11.0$ & II & & II & $12.923 \pm 0.012$ & 0.042 & $\mathrm{~V}$ & $\ldots$ & $12.666 \pm 0.016$ & 0.052 & $\mathrm{~V}$ & $\ldots$ & 0.23 & $\mathrm{~N}$ \\
\hline IC1396A-53 & $21: 37: 09.36+57: 29: 48.3$ & II & & II & $10.973 \pm 0.006$ & 0.019 & $\mathrm{~V}$ & $\ldots$ & $10.515 \pm 0.009$ & 0.028 & $\mathrm{~V}$ & $\ldots$ & 0.74 & $\mathrm{~V}$ \\
\hline IC1396A-54 & $21: 37: 09.44+57: 30: 36.7$ & II & & II & $12.125 \pm 0.009$ & 0.035 & $\mathrm{~V}$ & $\ldots$ & $11.817 \pm 0.009$ & 0.047 & $\mathrm{~V}$ & $\ldots$ & 0.72 & $\mathrm{~V}$ \\
\hline IC1396A-55 & $21: 37: 10.13+57: 31: 26.6$ & II & & II & $13.252 \pm 0.015$ & 0.029 & $\mathrm{~N}$ & $\ldots$ & $12.918 \pm 0.019$ & 0.029 & $\mathrm{~N}$ & $\ldots$ & 0.24 & $\mathrm{~N}$ \\
\hline IC1396A-56 & $21: 37: 10.31+57: 30: 18.9$ & II & & II & $11.545 \pm 0.009$ & 0.011 & $\mathrm{~N}$ & $\ldots$ & $11.417 \pm 0.009$ & 0.017 & $\mathrm{~N}$ & $\ldots$ & 0.17 & $\mathrm{~N}$ \\
\hline IC1396A-57 & $21: 37: 10.54+57: 31: 12.4$ & II & & II & $11.130 \pm 0.006$ & 0.058 & $\mathrm{~V}$ & $\ldots$ & $10.724 \pm 0.009$ & 0.063 & $\mathrm{~V}$ & $\ldots$ & 2.19 & $\mathrm{~V}$ \\
\hline IC1396A-58 & $21: 36: 56.51+57: 31: 51.6$ & $\ldots$ & $\kappa, \mathrm{II}$ & II & $12.323 \pm 0.009$ & 0.016 & $\mathrm{~N}$ & $\ldots$ & $11.626 \pm 0.009$ & 0.015 & $\mathrm{~N}$ & $\ldots$ & 0.05 & $\mathrm{~N}$ \\
\hline IC1396A-59 & $21: 37: 03.04+57: 30: 48.7$ & $\ldots$ & $\mu, \mathrm{I}$ & I & $13.564 \pm 0.016$ & 0.032 & $\mathrm{~N}$ & $\ldots$ & $12.218 \pm 0.013$ & 0.036 & $\mathrm{~N}$ & $\ldots$ & 0.48 & $\mathrm{~N}$ \\
\hline IC1396A-60 & $21: 36: 47.18+57: 29: 52.6$ & $\ldots$ & & II & $10.406 \pm 0.006$ & 0.062 & $\mathrm{~V}$ & $\ldots$ & $9.869 \pm 0.009$ & 0.04 & $\mathrm{~V}$ & $\ldots$ & 1.83 & V \\
\hline IC1396A-61 & $21: 36: 47.63+57: 29: 54.1$ & $\ldots$ & & II & $10.416 \pm 0.006$ & 0.030 & V & $\ldots$ & $9.960 \pm 0.009$ & 0.033 & $\mathrm{~V}$ & $\ldots$ & 1.30 & $\mathrm{~V}$ \\
\hline IC1396A-62 & $21: 37: 14.51+57: 28: 40.9$ & $\ldots$ & & II & $11.833 \pm 0.009$ & 0.061 & $\mathrm{~V}$ & $\ldots$ & $\ldots$ & $\ldots$ & $\ldots$ & $\ldots$ & 1.96 & V \\
\hline IC1396A-63 & $21: 37: 17.42+57: 29: 27.5$ & $\ldots$ & & II & $11.835 \pm 0.009$ & 0.038 & $\mathrm{~V}$ & $\ldots$ & $\ldots$ & $\ldots$ & $\ldots$ & $\ldots$ & 1.01 & V \\
\hline IC1396A-64 & $21: 37: 17.37+57: 29: 20.8$ & $\ldots$ & & II & $12.886 \pm 0.012$ & 0.019 & $\mathrm{~N}$ & $\ldots$ & $\ldots$ & $\ldots$ & $\ldots$ & $\ldots$ & 0.10 & $\mathrm{~N}$ \\
\hline IC1396A-65 & $21: 36: 42.49+57: 25: 23.3$ & $\ldots$ & & II & $13.176 \pm 0.015$ & 0.018 & $\mathrm{~N}$ & $\ldots$ & $12.943 \pm 0.019$ & 0.036 & $\mathrm{~N}$ & $\ldots$ & 0.16 & $\mathrm{~N}$ \\
\hline IC1396A-66 & $21: 37: 05.87+57: 32: 12.5$ & $\ldots$ & & II & $13.362 \pm 0.015$ & 0.044 & $\mathrm{~V}$ & 9 & $13.074 \pm 0.021$ & 0.033 & $\mathrm{~N}$ & $\ldots$ & 0.25 & V \\
\hline IC1396A-67 & $21: 35: 58.52+57: 29: 15.1$ & $\ldots$ & & II & $13.546 \pm 0.016$ & 0.036 & $\mathrm{~N}$ & $\ldots$ & $13.098 \pm 0.021$ & 0.027 & $\mathrm{~N}$ & $\ldots$ & 0.15 & $\mathrm{~N}$ \\
\hline IC1396A-68 & $21: 36: 56.26+57: 29: 52.3$ & $\ldots$ & & II & $13.551 \pm 0.016$ & 0.023 & $\mathrm{~N}$ & $\ldots$ & $12.974 \pm 0.02$ & 0.024 & $\mathrm{~N}$ & $\ldots$ & 0.09 & $\mathrm{~N}$ \\
\hline IC1396A-69 & $21: 36: 38.03+57: 26: 57.9$ & $\ldots$ & & II & $13.578 \pm 0.016$ & 0.048 & $\mathrm{~V}$ & $\ldots$ & $13.172 \pm 0.022$ & 0.077 & $\mathrm{~V}$ & $\ldots$ & 0.90 & $\mathrm{~V}$ \\
\hline IC1396A-70 & $21: 36: 12.60+57: 31: 26.3$ & $\ldots$ & & II & $13.997 \pm 0.021$ & 0.126 & $\mathrm{~V}$ & $\ldots$ & $13.473 \pm 0.026$ & 0.095 & $\mathrm{~V}$ & $\ldots$ & 1.08 & $\mathrm{~V}$ \\
\hline IC1396A-71 & $21: 36: 40.34+57: 25: 45.7$ & $\ldots$ & & II & $14.038 \pm 0.022$ & 0.043 & $\mathrm{~N}$ & $\ldots$ & $13.617 \pm 0.028$ & 0.042 & $\mathrm{~N}$ & $\ldots$ & 0.34 & $\mathrm{~N}$ \\
\hline IC1396A-72 & $21: 36: 18.97+57: 29: 05.1$ & $\ldots$ & & I & $14.186 \pm 0.024$ & 0.051 & $\mathrm{~V}$ & $\ldots$ & $\ldots$ & $\ldots$ & $\ldots$ & $\ldots$ & 0.13 & $\mathrm{~N}$ \\
\hline IC1396A-73 & $21: 37: 11.78+57: 30: 34.9$ & $\ldots$ & & II & $14.269 \pm 0.026$ & 0.051 & $\mathrm{~N}$ & $\ldots$ & $13.823 \pm 0.031$ & 0.051 & $\mathrm{~N}$ & $\ldots$ & 0.26 & $\mathrm{~N}$ \\
\hline IC1396A-74 & $21: 36: 36.35+57: 32: 09.3$ & $\ldots$ & & I & $15.491 \pm 0.059$ & 0.053 & $\mathrm{~N}$ & $\ldots$ & $14.663 \pm 0.050$ & 0.040 & $\mathrm{~N}$ & $\ldots$ & -0.05 & $\mathrm{~V}$ \\
\hline
\end{tabular}

Notes.

a Objects are named following the order in Table 6 of Sicilia-Aguilar et al. (2006; note that not all their members are included), objects -58 and -59 are YSOs from Reach et al. (2004) not in common with Sicilia-Aguilar et al. (2006), and objects from -60 to -74 are our new candidate members from Table 1.

${ }^{b}$ YSO Class as in Sicilia-Aguilar et al. (2006).

${ }^{\mathrm{c}}$ ID and YSO Class as in Reach et al. (2004).

d YSO Class in this paper. Members in Sicilia-Aguilar et al. (2006) that do not show IR excess in our data have been classified as Class III.

e Classification in disagreement with Sicilia-Aguilar et al. (2006).

${ }^{\mathrm{f}}$ Uncertain cluster members following Sicilia-Aguilar et al. (2006). 

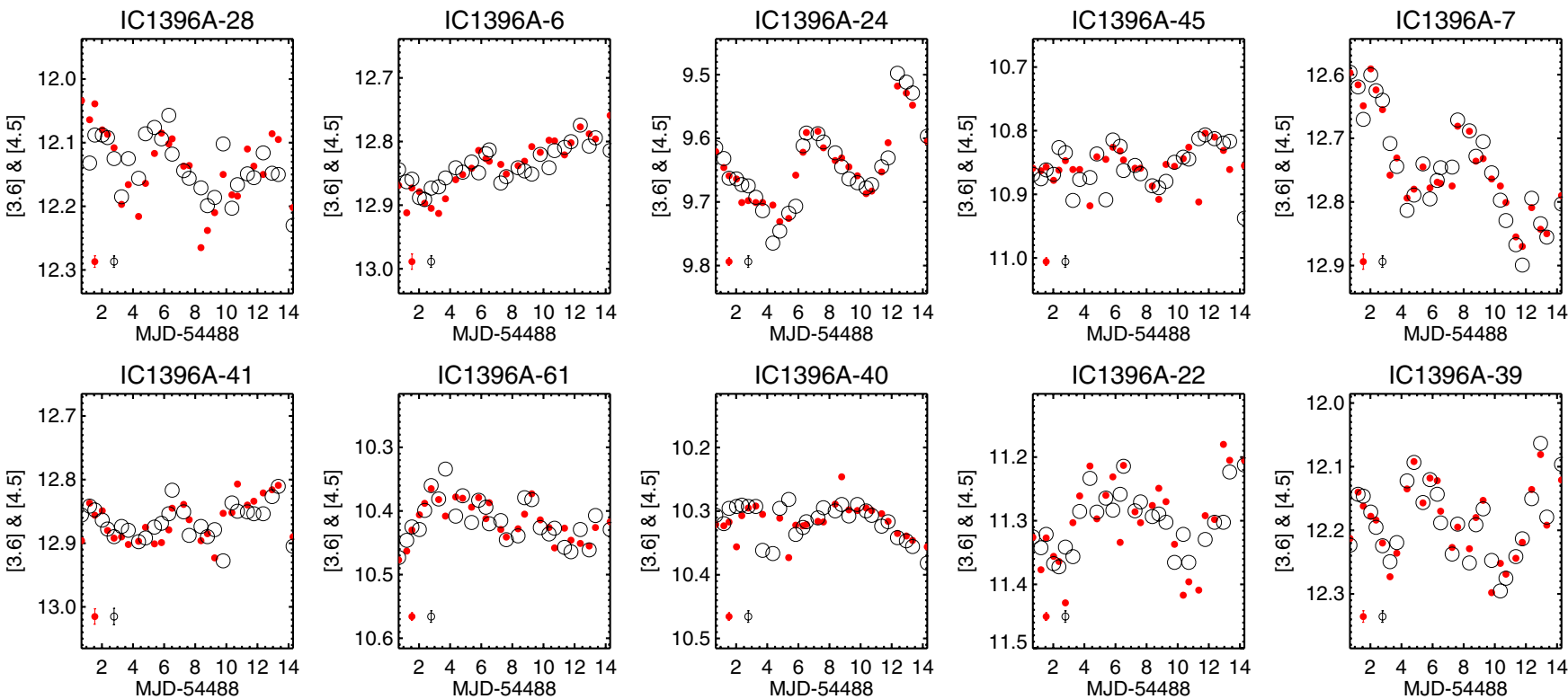

IC1396A-17

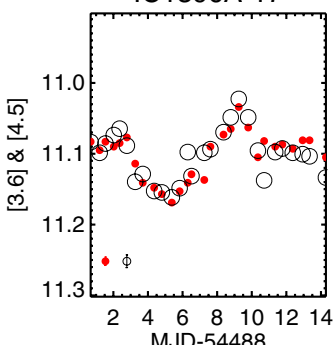

IC1396A-29

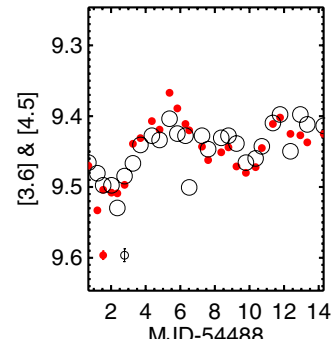

IC1396A-13

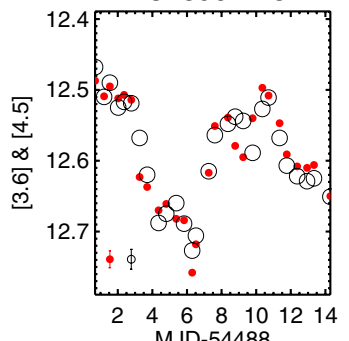

IC1396A-37
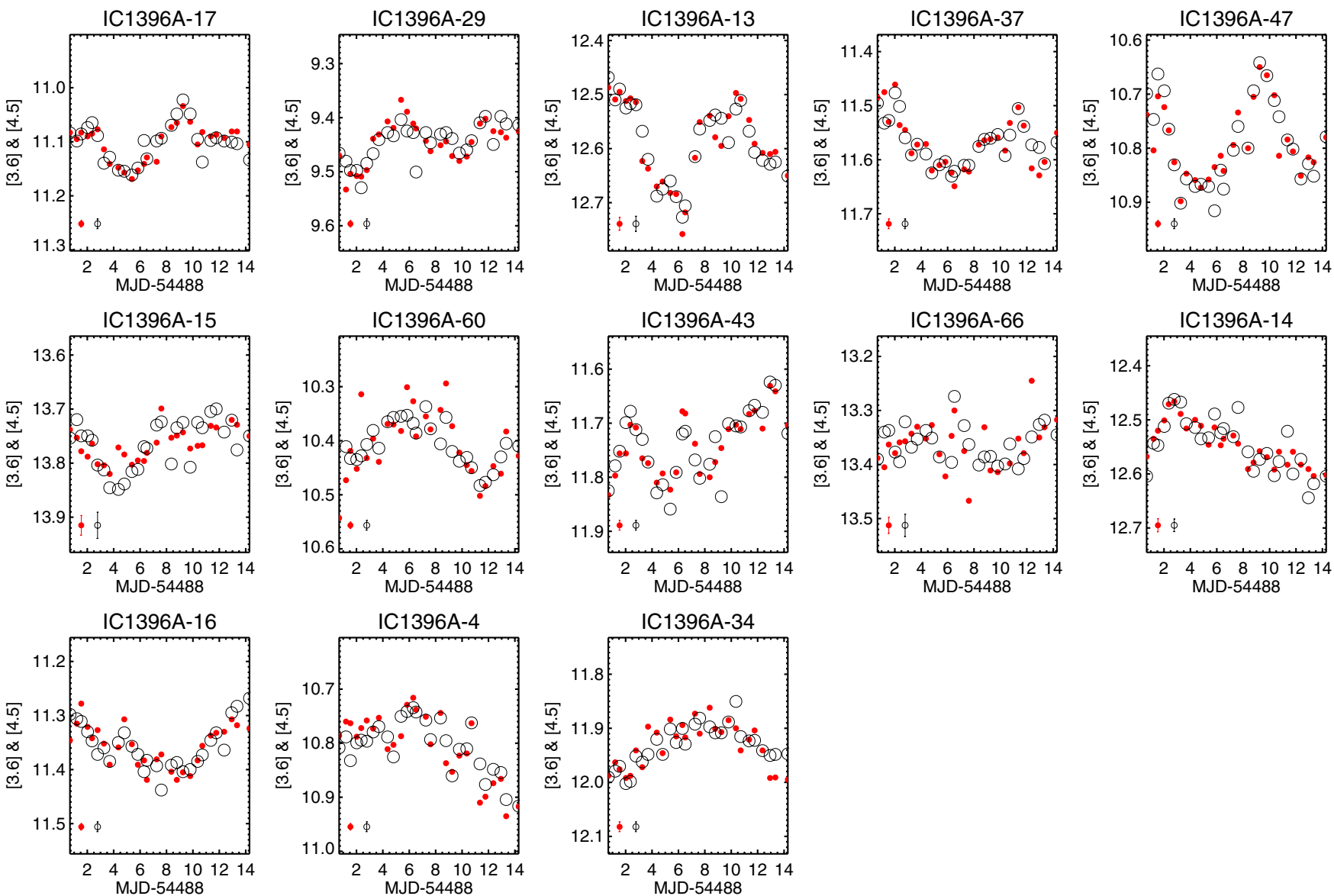

Figure 8. Light curves for the 18 IC1396A YSOs which present periodic-like, colorless variations. In this figure, we show objects that seem to have increasing periods (from 5 to more than 14 days) from the top left panel to the right bottom one. Red filled circles represent the Ch. 1 data while open black circles show the Ch. 2 data. The Ch. 2 light curves have been shifted in the $y$-axis to match the mean magnitudes in Ch. 1 .

(A color version of this figure is available in the online journal.)

an apparently significant decrease in flux over a several day period at $8 \mu \mathrm{m}$ (Figure 11(b)). Objects 35 and 57 are Class II stars and object 74 is a Class I YSO. Possible causes: radially differential heating of the inner disk; obscuration by an inner disk overdensity.

\subsection{The Staring Data: Short-term Variable Members}

Thirty eight out of the 42 targets that fall inside the FOV of Ch. 2 and Ch. 4 in the staring mode observations have enough good photometry to derive their time series, at least in one of 

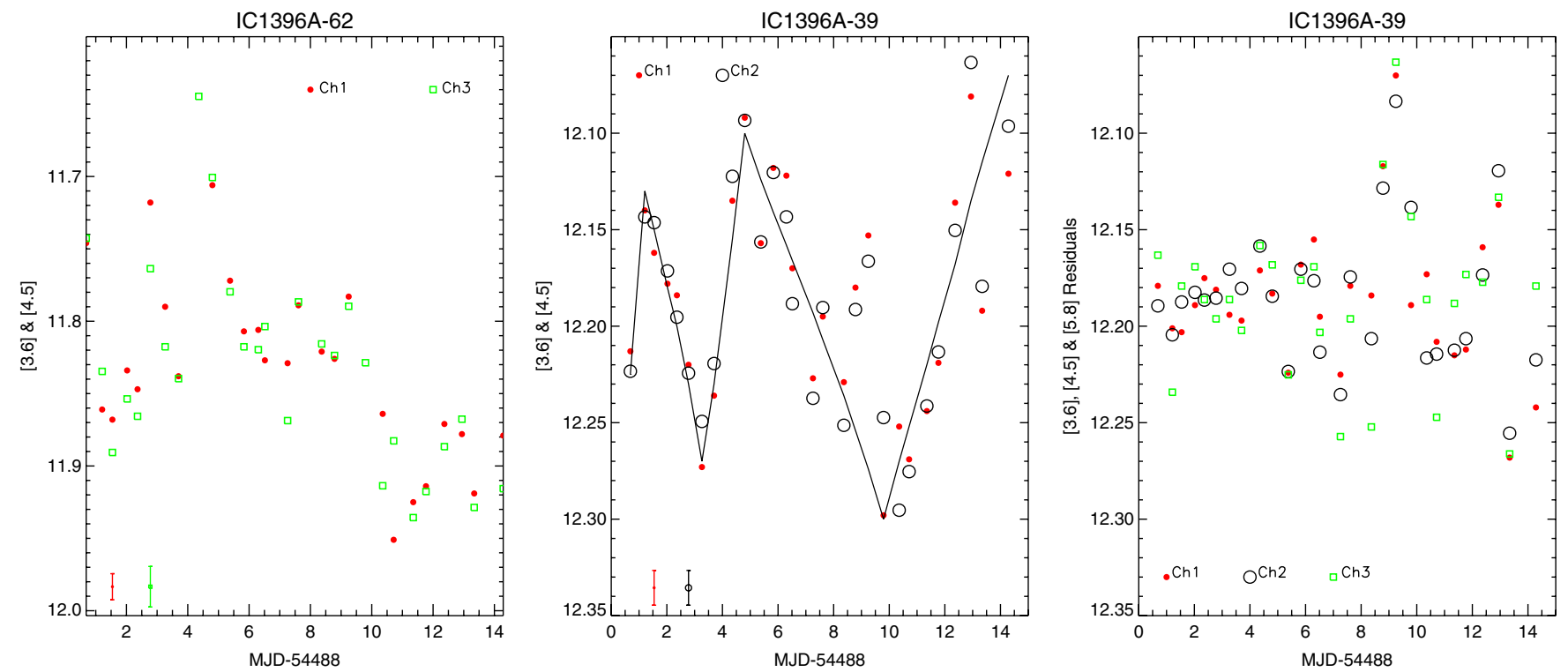

Figure 9. (a) Light curves for IC1396A-62. This object shows a sudden change in brightness of about 0.2 mag that lasts for 2 days. The object is located just at the border of Ch. 2 and Ch. 4 FOV and thus the light curves presented are those for Ch. 1 (filled circles) and Ch. 3 (open squares). (b) Light curves for IC1396A-39, Ch. 1 (red dots), Ch. 2 (black circles), with a saw-tooth pattern illustrating the long-term variation. (c) Residuals from the light curve in (b) after subtracting the saw-tooth pattern showing a flare or accretion event. Ch. 3 data are also included (open squares).

(A color version of this figure is available in the online journal.)

Table 3

Time Series in the Four IRAC Bands for the Variable YSOs of Our Sample

\begin{tabular}{cccccr}
\hline \hline Object & MJD (days) & {$[3.6] \mathrm{e}[3.6]$} & {$[4.5] \mathrm{e}[4.5]$} & {$[5.8] \mathrm{e}[5.8]$} & {$[8.0] \mathrm{e}[8.0]$} \\
\hline IC1396A-1 & 54488.6878678 & 13.2330 .018 & 12.5960 .013 & 11.8330 .023 & 10.4480 .042 \\
IC1396A-1 & 54489.2077075 & 13.1490 .014 & 12.5450 .012 & 11.7410 .028 & 10.3650 .037 \\
IC1396A-1 & 54489.5421136 & 13.1780 .019 & 12.5290 .011 & 11.7300 .020 & 10.4150 .038 \\
IC1396A-1 & 54490.0224986 & 13.2290 .019 & 12.5830 .012 & 11.8420 .026 & 10.4050 .037 \\
IC1396A-1 & 54490.3616676 & 13.2120 .020 & 12.5720 .012 & 11.7600 .019 & 10.3600 .035 \\
IC1396A-1 & 54490.7798051 & 13.2560 .021 & 12.5850 .011 & 11.8000 .024 & 10.3400 .035 \\
IC1396A-1 & 54491.2620497 & 13.2930 .021 & 12.6820 .014 & 11.8670 .024 & 10.5010 .036 \\
IC1396A-1 & 54491.6974542 & 13.3840 .023 & 12.7740 .014 & 12.0000 .030 & 10.5310 .040 \\
IC1396A-1 & 54492.3575716 & 13.4280 .027 & 12.8360 .014 & 12.0650 .030 & 10.6040 .036 \\
IC1396A-1 & 54492.8026341 & 13.4240 .028 & 12.7830 .013 & 12.0370 .030 & 10.6330 .039 \\
\hline
\end{tabular}

(This table is available in its entirety in a machine-readable form in the online journal. A portion is shown here for guidance regarding its form and content.)

the two band passes. The remaining objects were saturated in our images (IC1396A-19 and IC1396A-30) or were too faint to obtain their photometry (IC1396A-26 and IC1396A-27). Five out of these 38 objects are new candidate members based on our new deep photometry. Table 5 presents the results of all targets including IRAC magnitudes, rms amplitudes for the light curves, result of the $\chi^{2}$ test, the period of the modulation observed if there is any, the $J$ variability index, and our final tag of variability. Fourteen objects were labeled by the $\chi^{2}$ statistic as variable in Ch. 2, however only four of them were labeled as variable in both band passes, IC1396A-35, IC1396A-47, IC1396A-57, and IC1396A-61 (Figure 13 shows the light curves of the shortterm variable stars). Another seven objects were labeled as variable by the $J$ variability index. The most interesting object is IC1396A-47, which shows a period of $3.4 \mathrm{hr}$ in both band passes (we discuss this star in more detail in Section 6.4). A few of the objects labeled as variable in Ch. 2, but not in Ch. 4 by the $\chi^{2}$ test, have light curves in Ch. 4 which follow the same trend and have similar amplitude to that of the light curve in Ch. 2. They have not been labeled as variable at Ch. 4, probably due to a much higher noise at $8.0 \mu \mathrm{m}$ than at $4.5 \mu \mathrm{m}$.
These objects are IC1396A-22, IC1396A-24, IC1396A-39, and IC1396A-43 - two of them are variable based on the $J$ index and the other two are just below the adopted $J$ index limit for variable stars. We consider the four of them as variable objects due to the shape similarity between both bandpasses. The remaining objects show amplitudes in Ch. 2 of order of the noise seen in the light curves in Ch. 4, not allowing the detection of variability in the latter channel. There is only one object, IC1396A-25, which has detected variability based on the $\chi^{2}$ test only in Ch. 4 . This object is variable based on the $J$ index however, it is very bright at $8 \mu \mathrm{m}$ and since latent buildup seems to be flux dependent, it is possible that our correction is not good enough for this object and the variability is instead spurious. The four objects with detected variability in both bandpasses show the same lightcurve shape in each band, IC1396A-35 and IC1326A-61 show a downward trend during the $7.5 \mathrm{hr}$ of observation, and IC1396A47 presents a pulsation-like variation (see Section 6.4). The last object labeled as variable in both channels, IC1396A-57, shows $\chi^{2}$ values that are only slightly above our detection threshold. Its light curves show a low-amplitude correlated variation and it is labeled as variable by the $J$ index. IC1396A-57 does vary on 

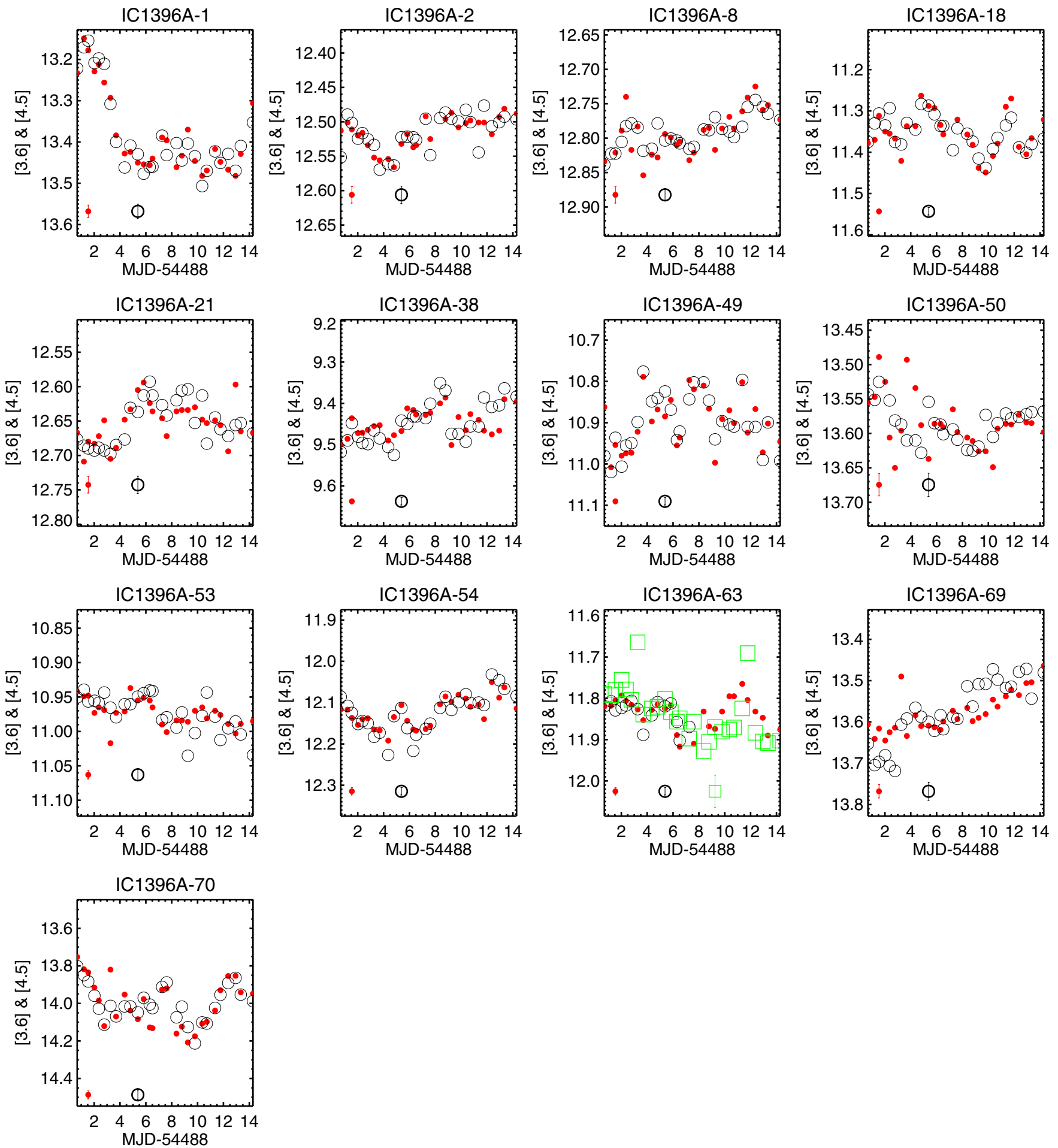

Figure 10. Light curves for the 18 IC1396A YSOs which show slow, nonperiodic colorless variability. In this figure, Ch. 1 (red filled circles) and Ch. 2 are plotted (black open circles). For object 63 the Ch. 3 data-green open squares-are also plotted.

(A color version of this figure is available in the online journal.)

long timescales, as shown in Figure 11. Finally, there are two objects which lack good photometry in Ch. 4-IC1396A-54 and IC1396A-60-but which show an upward trend in their Ch. 2 light curves. Since we cannot confirm the variability with the Ch. 4 data we do not include those objects in the final list of variable YSOs. Their light curves can be seen in Figure 14.

To summarize, eight out of 38 YSOs show correlated shortterm variability in Ch. 2 and Ch. 4 . All these objects also show long-term variability (see Section 5.1) and the variations seen in the $7.5 \mathrm{hr}$ period are consistent with the light-curve shapes seen in the mapping mode data (note that the staring observations were performed $5 \mathrm{hr}$ after the last mapping observation was finished).

The remaining objects are cataloged as nonvariable in short timescales and any possible variability has to be under the rms amplitudes quoted in Table 5. 

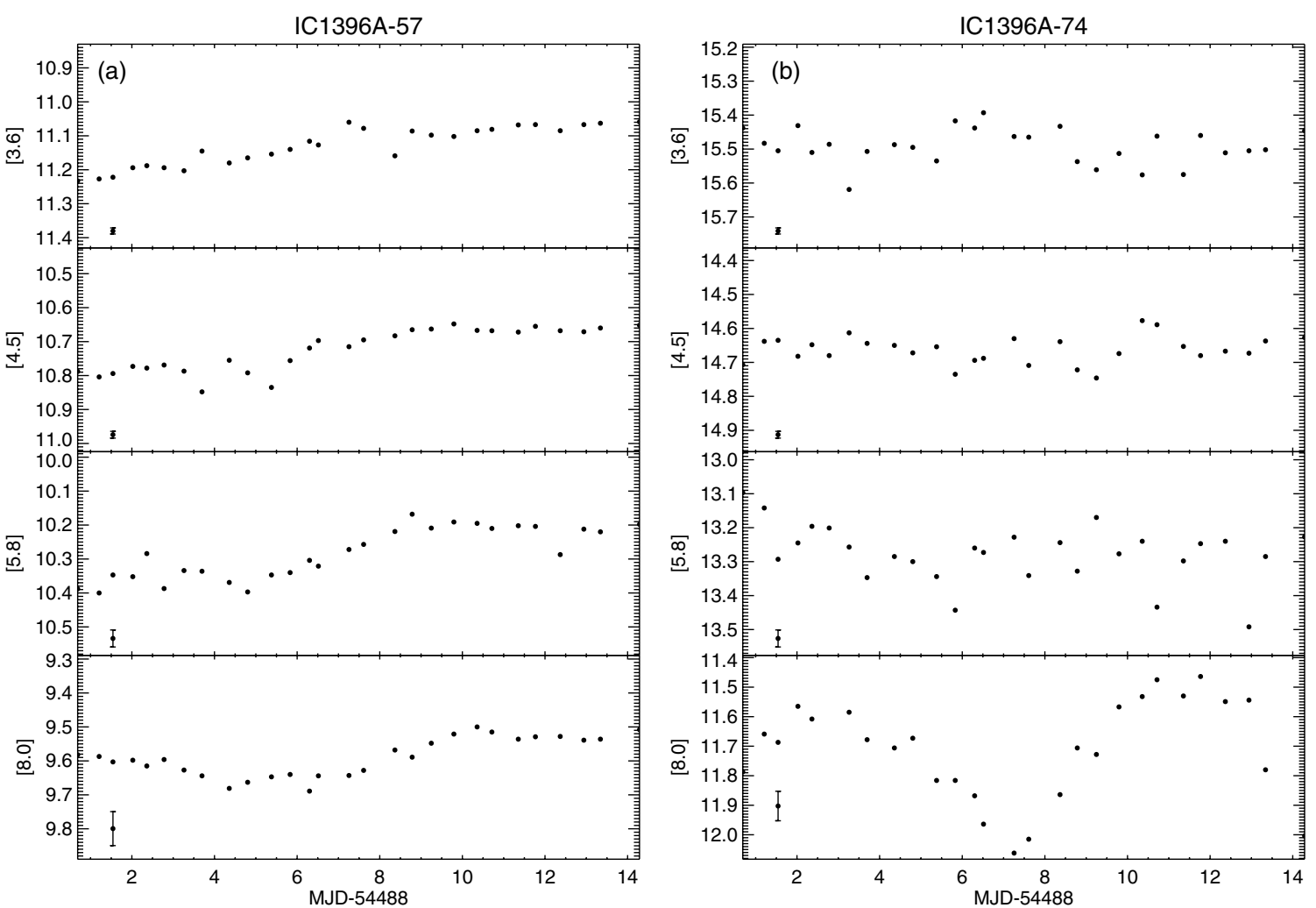

Figure 11. (a) Light curves for IC1396A-57. This object shows a continuous change in shape from Ch. 1 to Ch. 4 . The peak-to-peak amplitude at Ch. 4 is $\sim 0.2$ mag. (b) Light curves for IC1396A-74. The panels, from the top to the bottom, show the IRAC photometry at Ch. $1(3.6 \mu \mathrm{m}), \mathrm{Ch} .2(4.5 \mu \mathrm{m})$, Ch. $3(5.6 \mu \mathrm{m})$, and Ch. $4(8.0 \mu \mathrm{m})$, respectively. This object shows almost constant light curves at the three bluer bandpasses while the star fades in brightness in Ch. 4 for $\sim 6$ days. The amplitude of the variation is $\sim 0.5 \mathrm{mag}$.
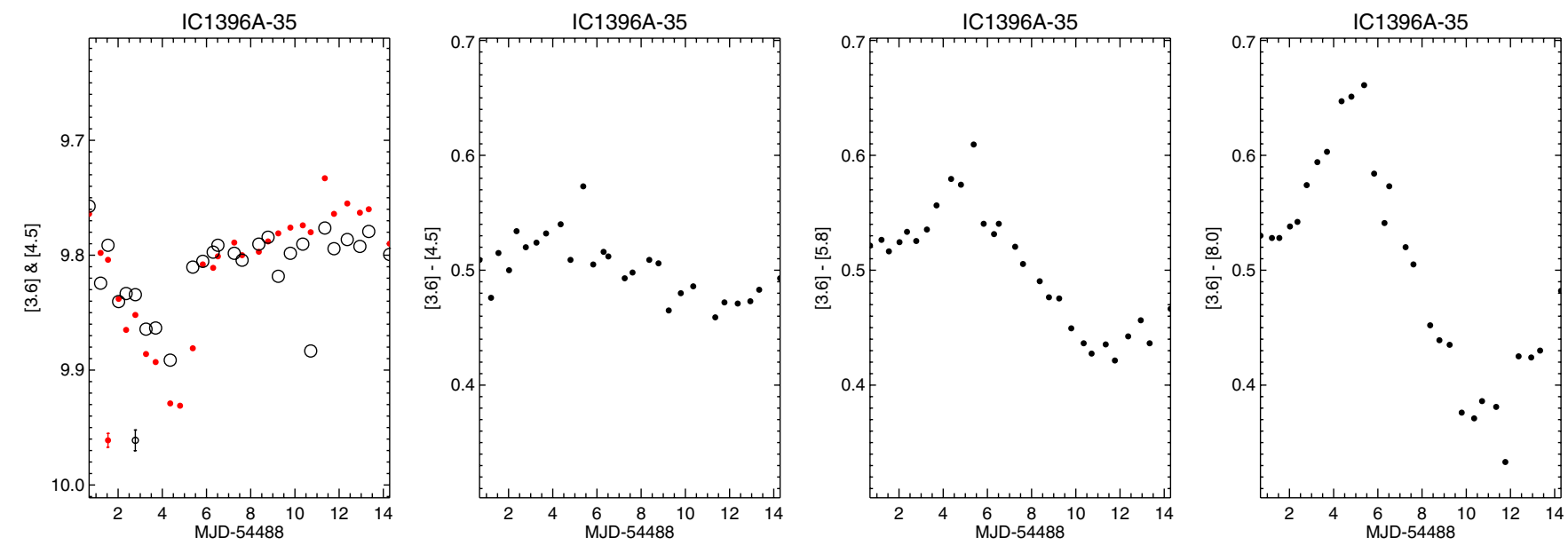

Figure 12. Light curves for IC1396A-35. Magnitude change with time in the rightmost diagram and changes of colors with time in the remaining diagrams. The star presents a decrease in brightness at the beginning of the observation, probably due to some obscuration process, and 4 days later the light curve becomes constant. The peak-to-peak amplitude in the color variation increases from $0.05 \mathrm{mag}$ in [3.6]-[4.5] to $0.3 \mathrm{mag}$ in [3.6]-[8.0].

(A color version of this figure is available in the online journal.)

\subsection{Variability of Stars Without IR Excesses in the Mapping Data}

In addition to the previously known members we have inspected the "field" (which includes both true field stars and YSOs lacking IR excesses) stars, in search of additional variable stars. Due to their lack of infrared excess, Class III YSOs cannot be separated from field objects at Spitzer wavelengths. However, given that photometric variability is one of the original defining characteristics of pre-main-sequence stars, the detection of variability can be used as a possible indicator of youth. Thus, we applied the $\chi^{2}$ and $J$ statistics to the entire sample of detected objects in Ch. 1 and Ch. 2. Six percent of the sample was labeled as variable in both tests. We visually inspected the light curves of all these objects to remove spurious variability detections due to bad photometric measurements or very noisy light curves. We identify 46 objects as being variable both in the visual inspection and in the statistical tests. Most of the light curves of these objects are consistent with rotational modulation with periods of the order of 4 to $>10$ days. These objects 

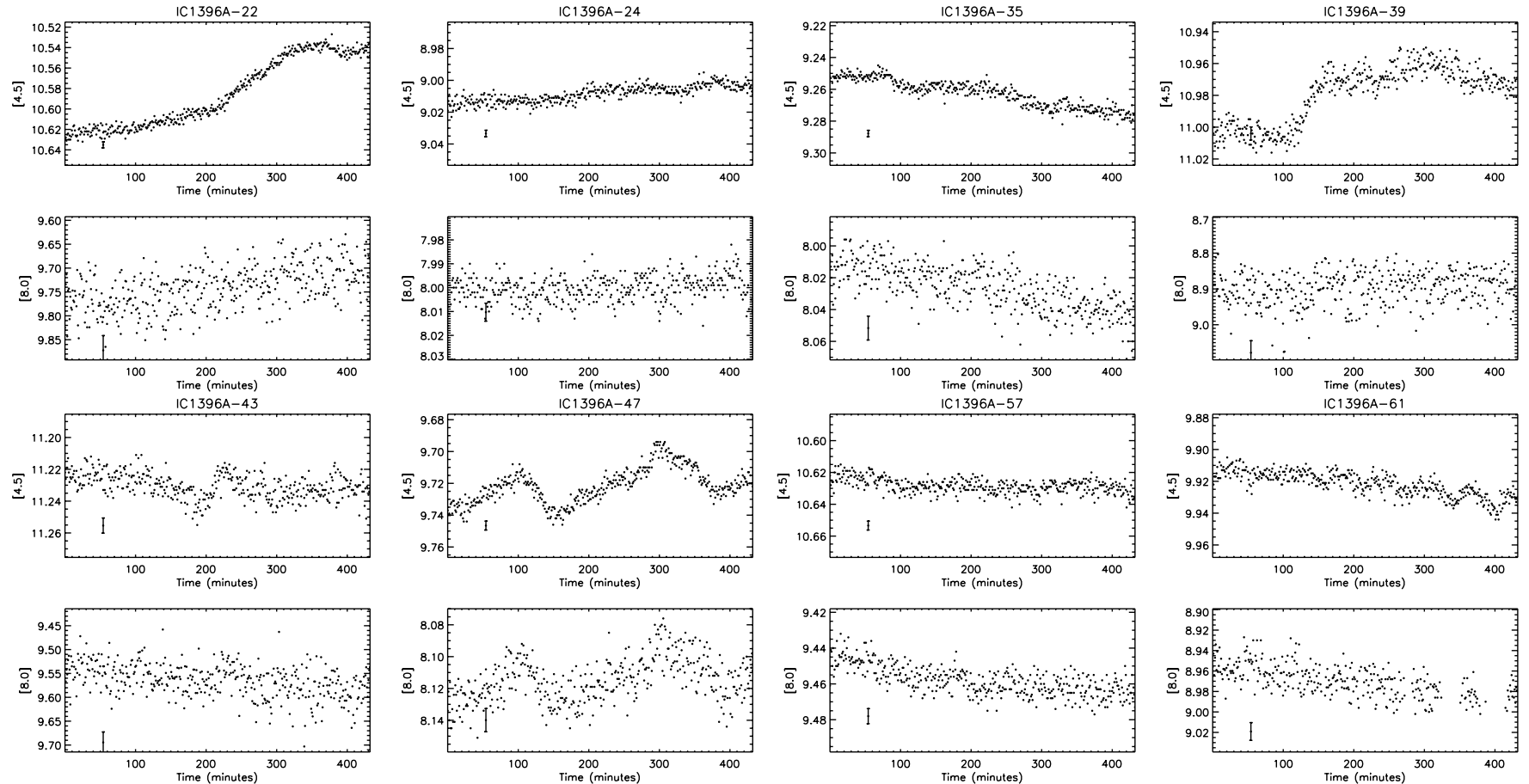

Figure 13. Light curves for the eight IC 1393A short-term variable YSOs. The top and bottom panels show Ch. 2 and Ch. 4 time series, respectively. The rms uncertainty of a single point is represented in the lower left corner of each panel. Note the different scales in the $y$-axis in the upper and lower panels.

Table 4

Peak-to-Peak Amplitudes for the 18 IC 1396A YSOs Which Present Periodic-like, Colorless Variations ${ }^{\mathrm{a}}$

\begin{tabular}{lcccc}
\hline \hline \multicolumn{1}{c}{ Object } & \multicolumn{4}{c}{ Peak-to-Peak Amplitudes (mag) } \\
\cline { 2 - 5 } & $3.6 \mu \mathrm{m}$ & $4.5 \mu \mathrm{m}$ & $5.8 \mu \mathrm{m}$ & $8.0 \mu \mathrm{m}$ \\
\hline IC 1396A-4 & 0.15 & 0.11 & 0.09 & 0.07 \\
IC 1396A-6 & 0.09 & 0.07 & 0.15 & $\ldots$ \\
IC 1396A-7 $^{\text {b }}$ & 0.19 & 0.19 & 0.17 & 0.15 \\
IC 1396A-13 $^{\mathrm{b}}$ & 0.17 & 0.17 & 0.23 & $\ldots$ \\
IC 1396A-14 $^{\text {c }}$ & 0.09 & 0.11 & 0.14 & 0.15 \\
IC 1396A-15 & 0.12 & 0.12 & 0.49 & 0.53 \\
IC 1396A-16 & 0.09 & 0.09 & 0.2 & 0.15 \\
IC 1396A-17 & 0.10 & 0.10 & 0.10 & 0.11 \\
IC 1396A-22 & 0.20 & 0.18 & 0.21 & 0.23 \\
IC 1396A-24 & 0.11 & 0.12 & 0.11 & 0.11 \\
IC 1396A-28c & 0.15 & 0.12 & 0.16 & 0.23 \\
IC 1396A-29 & 0.06 & 0.06 & 0.04 & 0.05 \\
IC 1396A-34 & 0.11 & 0.10 & 0.12 & 0.08 \\
IC 1396A-37 & 0.14 & 0.13 & 0.12 & 0.17 \\
IC 1396A-39 & 0.17 & 0.16 & 0.19 & 0.22 \\
IC 1396A-40 & 0.07 & 0.07 & 0.06 & 0.06 \\
IC 1396A-41 & 0.06 & 0.07 & 0.23 & 0.51 \\
IC 1396A-43 & 0.16 & 0.16 & 0.17 & 0.18 \\
IC 1396A-45 & 0.07 & 0.07 & 0.07 & 0.06 \\
IC 1396A-47 & 0.25 & 0.21 & 0.24 & 0.25 \\
IC 1396A-60 & 0.13 & 0.11 & 0.12 & 0.07 \\
IC 1396A-61 & 0.08 & 0.09 & 0.11 & 0.09 \\
IC 1396A-66 & 0.08 & 0.11 & 0.25 & 0.3 \\
\hline
\end{tabular}

Notes.

${ }^{a}$ Long-term data.

b This object is too faint to derive its amplitude at $8.0 \mu \mathrm{m}$.

${ }^{c}$ The light curves of this object are much noisier at 5.8 and $8.0 \mu \mathrm{m}$ than at shorter wavelengths.

present Rayleigh-Jeans-like SEDs with no infrared excess other than contamination expected from the nebulosity. While we
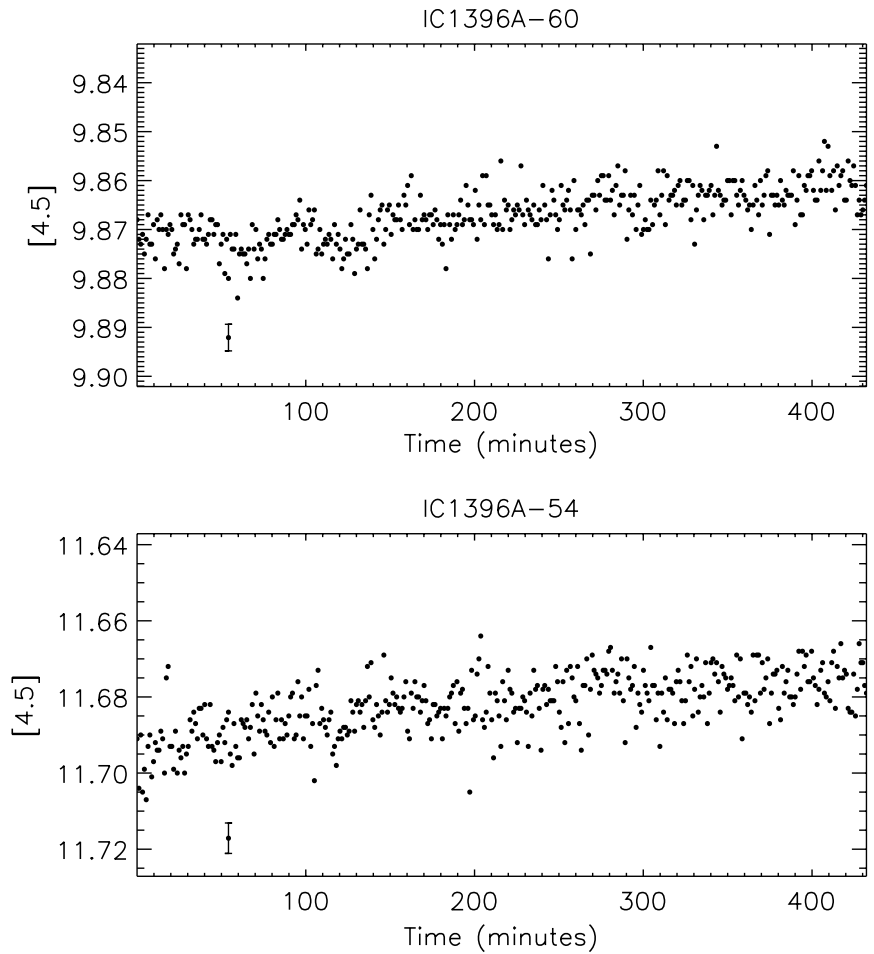

Figure 14. Ch. 2 light curves for the two IC 1393A YSOs, which lacking the Ch. 4 light curves, do show an upward trend in their Ch. 2 light curves. The rms uncertainty of a single point is represented in the lower left corner of each panel.

are reasonably convinced these stars are variable at 3.6 and $4.5 \mu \mathrm{m}$, without further data we cannot know whether they are members of IC1396A, members of the older Tr37 cluster, spotted field stars, or other variables of uncertain type. Data at other wavelengths are needed to categorize these stars. Light 
Table 5

Short-time Variability: Main Results from the Staring Data

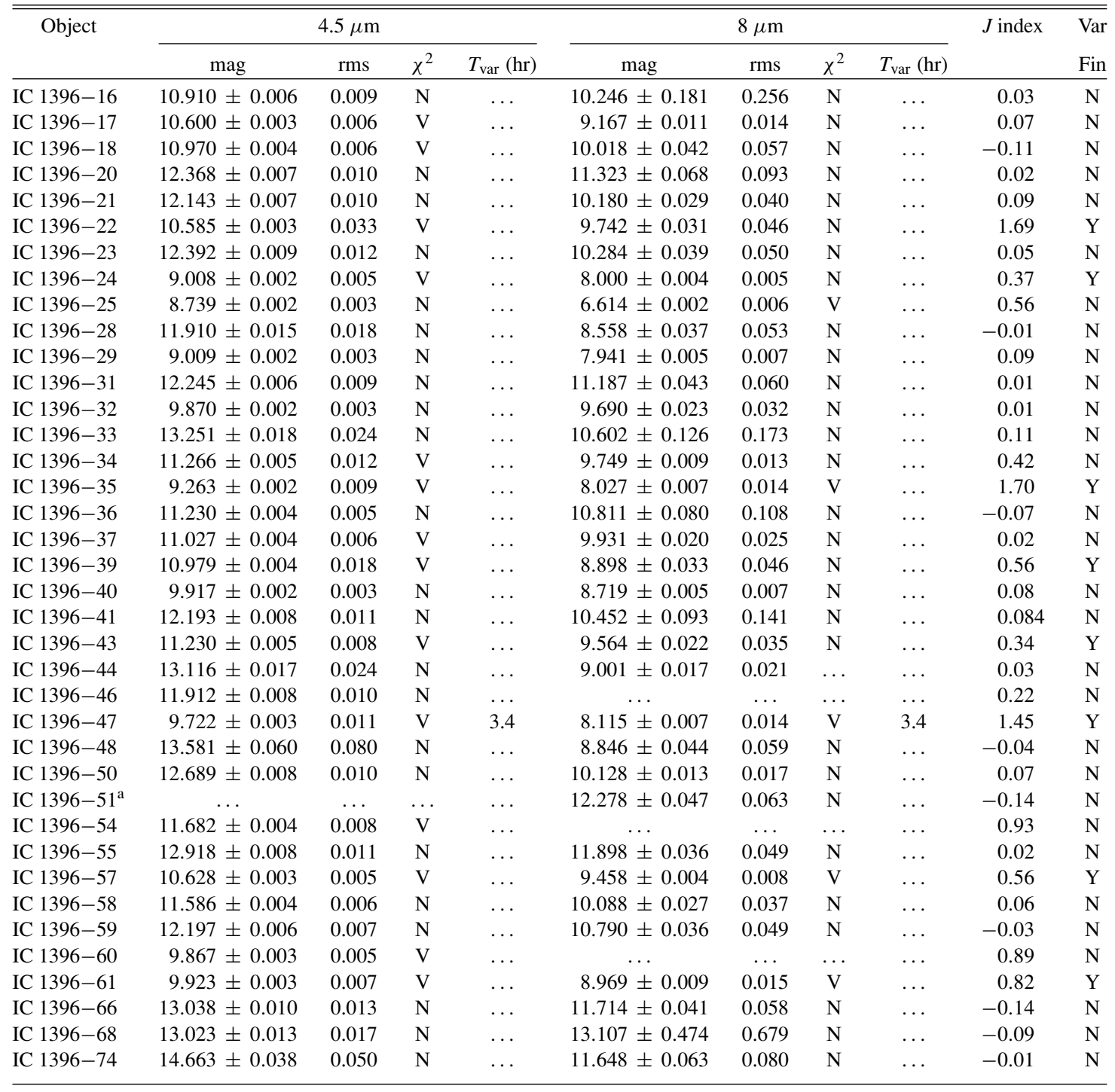

Note.

a The object is out of the FOV in Ch. 2 .

curves for a few of these new candidates can be seen in Figure 15 and their photometry is presented in Table 6.

\subsection{Properties of the Variable YSOs}

As summarized in Table 7, 41 out of the 69 objects that form our sample of YSOs (54 previously known YSOs plus 15 new candidates) are variable on long timescales. Forty percent of the 69 YSOs in Table 2 show peak-to-peak amplitudes of at least $0.1 \mathrm{mag}$ and $60 \%$ of the sample present peak-topeak amplitudes greater than 0.05 mag. Most of the variable YSOs show colorless variations (at least within the photometric errors) but there are three objects whose IRAC colors vary with time. In two out of them the object gets redder as it gets fainter, as would be expected if variable extinction or rotational modulation of a spotted photosphere is the cause of the photometric changes. Twenty three YSOs show what seems to be well defined apparently periodic variability with amplitudes up to about $0.2 \mathrm{mag}$. Where we are able to derive estimated periods, the periods range from about 5 days to about
11 days. These variables include nine Class I and 14 Class II objects.

In order to help determine the physical cause(s) for this variability, it is useful to determine if there is anything special about the variable stars-i.e., are they younger than average? Are they more embedded than average? Are they higher or lower mass than average? Figure 16 shows the spatial distribution of the different types of YSOs. The figure illustrates that all the Class I and most of the objects with periodic-like light curves are located within the most embedded areas of the nebulosity while the Class II stars seem to be more spread out across the mosaic. Figure 17 shows the IRAC [3.6]-[4.5] versus [5.8]-[8.0] diagram where the YSOs of different types have been identified (red open diamonds represent large amplitude variables-peakto-peak amplitudes of at least $0.1 \mathrm{mag}$ - while blue open circles stand for constant objects). The diagram shows that the large amplitude variables tend to be redder in the IRAC colors (though some other stars are also quite red and do not show significant variability with IRAC). We conclude from Figures 16 and 17 that while our large amplitude variables can be either Class I 
Table 6

Photometry for the Variable Objects Found in the Field

\begin{tabular}{|c|c|c|c|c|c|c|c|c|}
\hline $\mathrm{ID}^{\mathrm{a}}$ & R.A. (J2000), Decl. (J2000) & $J^{\mathrm{b}} \mathrm{e} J$ & $H^{\mathrm{b}} \mathrm{e} H$ & $K_{s}^{\mathrm{b}} \mathrm{e} K_{s}$ & [3.6] e[3.6] & {$[4.5] \mathrm{e}[4.5]$} & [5.8] e[5.8] & {$[8.0] \mathrm{e}[8.0]$} \\
\hline 347 & $21: 35: 51.08+57: 28: 12.4$ & 15.3810 .033 & 14.8810 .042 & 14.6110 .038 & 14.2720 .029 & 14.3220 .027 & 13.2680 .135 & 11.3310 .095 \\
\hline 751 & $21: 36: 15.20+57: 25: 27.8$ & 15.4420 .047 & 14.6590 .052 & 14.4180 .038 & 13.7870 .01 & 13.5560 .012 & 13.5850 .063 & 12.3650 .06 \\
\hline 823 & $21: 36: 07.44+57: 26: 43.4$ & $\ldots$ & $\ldots$ & $\ldots$ & 12.8690 .035 & 11.0320 .025 & 9.6350 .019 & 8.5760 .055 \\
\hline 1230 & $21: 35: 58.07+57: 28: 50.4$ & 15.5960 .088 & 15.0590 .125 & 14.8360 .091 & 13.8990 .034 & 14.0670 .055 & 13.8090 .18 & \\
\hline 1256 & $21: 35: 53.10+57: 29: 37.1$ & 15.2080 .031 & 14.7090 .047 & 14.4990 .036 & 14.4240 .025 & 14.4070 .027 & 15.2280 .417 & 14.5290 .908 \\
\hline 1469 & $21: 35: 55.39+57: 29: 42.8$ & 15.6540 .034 & 15.0590 .042 & 14.8570 .049 & 14.6880 .028 & 14.6730 .032 & 14.6030 .207 & $\ldots$ \\
\hline 1685 & $21: 35: 55.64+57: 30: 03.3$ & 15.3140 .03 & 14.7670 .041 & 14.4530 .038 & 14.1360 .014 & 14.1510 .019 & 14.0340 .087 & $\ldots$ \\
\hline 2519 & $21: 36: 01.65+57: 30: 50.0$ & 15.5980 .041 & 14.9570 .052 & 14.7010 .041 & 14.1460 .017 & 14.020 .018 & 14.0820 .137 & 13.7550 .269 \\
\hline 2712 & $21: 36: 10.97+57: 29: 50.7$ & 15.6490 .034 & 14.6010 .037 & 14.2560 .027 & 14.110 .019 & 14.0670 .022 & 14.2040 .319 & 13.2460 .835 \\
\hline 2812 & $21: 36: 40.34+57: 25: 45.6$ & 16.2920 .049 & 15.4390 .063 & 14.8620 .04 & 14.1020 .015 & 13.6880 .016 & 13.1870 .042 & 12.5060 .047 \\
\hline 3115 & $21: 36: 13.35+57: 30: 16.2$ & 15.6970 .039 & 15.1110 .052 & 14.8540 .05 & 14.7160 .039 & 14.6350 .035 & 14.7530 .342 & $\ldots$ \\
\hline 3154 & $21: 36: 17.04+57: 29: 48.2$ & 15.6740 .034 & 14.630 .041 & 14.1980 .029 & 13.7890 .048 & 13.7410 .053 & 14.6191 .782 & \\
\hline 3367 & $21: 36: 33.20+57: 27: 51.9$ & 14.9850 .028 & 14.4130 .04 & 14.2080 .031 & 14.0010 .022 & 13.8740 .018 & 14.1720 .088 & $\ldots$ \\
\hline 3402 & $21: 36: 16.14+57: 30: 26.9$ & 14.5980 .025 & 14.2090 .036 & 14.0060 .026 & 13.9090 .017 & 13.7970 .014 & 13.6350 .119 & 12.5490 .248 \\
\hline 3444 & $21: 36: 17.94+57: 30: 16.3$ & $15.531^{\mathrm{c}}-$ & 15.350 .07 & 15.0480 .057 & 14.4740 .034 & 14.4190 .033 & $\ldots$ & $\ldots$ \\
\hline 3526 & $21: 36: 45.86+57: 26: 22.9$ & 15.1740 .03 & 14.4940 .043 & 14.3390 .033 & 14.2640 .012 & 14.3260 .024 & 14.1710 .091 & 13.0710 .073 \\
\hline 3795 & $21: 36: 33.02+57: 28: 49.4$ & 16.1540 .048 & 14.9190 .046 & 14.1270 .032 & 13.7380 .04 & 13.3440 .037 & 12.8950 .354 & 12.8741 .697 \\
\hline 4459 & $21: 36: 36.94+57: 29: 28.6$ & 15.7150 .03 & 15.0140 .04 & 14.5990 .041 & 14.2670 .076 & 14.1730 .11 & 12.5560 .322 & $\ldots$ \\
\hline 4535 & $21: 36: 37.58+57: 29: 31.7$ & 15.4680 .03 & 14.9830 .038 & 14.8160 .045 & 14.4440 .078 & 14.4310 .105 & 12.9780 .176 & 11.4330 .221 \\
\hline 4780 & $21: 36: 54.72+57: 27: 26.7$ & $\ldots$ & $\ldots$ & $\ldots$ & 16.9990 .109 & 16.8360 .255 & $\ldots$ & $\ldots$ \\
\hline 5053 & $21: 36: 12.99+57: 34: 05.4$ & 15.1670 .03 & 14.0530 .032 & 13.7520 .024 & 13.4740 .022 & 13.4980 .018 & 13.3550 .161 & 13.580 .606 \\
\hline 5120 & $21: 36: 38.61+57: 30: 27.0$ & 17.1130 .103 & 15.5280 .051 & 14.9370 .051 & 14.8730 .134 & 14.7080 .145 & $\ldots$ & $\ldots$ \\
\hline 5272 & $21: 36: 40.49+57: 30: 25.8$ & 15.990 .052 & 14.5240 .053 & $13.664^{\mathrm{c}} \ldots$ & 13.320 .043 & 13.2250 .061 & 12.120 .116 & 10.6380 .172 \\
\hline 5290 & $21: 36: 28.43+57: 32: 13.5$ & 14.4310 .023 & 13.4820 .03 & 13.1440 .019 & 12.9010 .013 & 12.8240 .012 & 13.8890 .321 & $\ldots$ \\
\hline 5546 & $21: 36: 47.62+57: 29: 54.0$ & 13.4970 .024 & 12.3560 .038 & 11.5930 .019 & 10.4770 .004 & 10.0170 .004 & 9.6720 .012 & 9.0820 .036 \\
\hline 5580 & $21: 36: 25.98+57: 33: 10.2$ & 14.8830 .029 & 14.2650 .043 & 14.0110 .029 & 13.7020 .037 & 13.6380 .025 & 14.1960 .629 & $\ldots$ \\
\hline 5610 & $21: 36: 49.03+57: 29: 49.2$ & 16.9040 .079 & 15.510 .059 & 14.6480 .043 & 14.1570 .043 & 14.1260 .072 & 15.4892 .214 & $\ldots$ \\
\hline 5647 & $21: 36: 44.11+57: 30: 38.3$ & 16.2140 .056 & 15.2370 .06 & 14.7540 .045 & 14.4310 .085 & 14.4310 .075 & 14.0230 .386 & $\ldots$ \\
\hline 5676 & $21: 36: 44.71+57: 30: 37.4$ & 16.4450 .053 & 15.4640 .063 & 14.8510 .043 & 14.3880 .097 & 14.5010 .094 & $\ldots$ & 11.1270 .166 \\
\hline 5680 & $21: 36: 16.09+57: 34: 48.6$ & 11.970 .022 & 11.1850 .028 & 10.9310 .016 & 10.7910 .004 & 10.7460 .004 & 10.770 .01 & 10.6840 .01 \\
\hline 5998 & $21: 36: 45.86+57: 31: 03.5$ & $\ldots$ & $\ldots$ & $\ldots$ & 14.2230 .106 & 14.5780 .146 & $\ldots$ & 10.1660 .294 \\
\hline 6200 & $21: 36: 53.17+57: 30: 18.7$ & $17.607^{\mathrm{c}}-$ & 16.3150 .123 & 15.1770 .067 & 14.4260 .073 & 14.1180 .056 & 14.51 .015 & $\ldots$ \\
\hline 6450 & $21: 36: 36.50+57: 33: 14.3$ & 16.8760 .079 & 16.1960 .125 & 15.7810 .092 & 14.8150 .116 & 14.9330 .098 & $\ldots$ & $\ldots$ \\
\hline 6710 & $21: 36: 34.86+57: 33: 57.1$ & 15.1350 .026 & 14.4780 .031 & 14.2510 .031 & 14.1710 .016 & 14.1750 .021 & 14.0630 .087 & $\ldots$ \\
\hline 6975 & $21: 36: 25.61+57: 35: 46.2$ & $\ldots$ & $\ldots$ & $\ldots$ & 14.1520 .058 & 14.0680 .051 & 14.5860 .365 & $\ldots$ \\
\hline 7003 & $21: 36: 52.64+57: 31: 50.4$ & 15.0050 .029 & 13.7030 .032 & 13.1560 .019 & 12.6730 .024 & 12.6230 .016 & 11.8160 .115 & 10.260 .157 \\
\hline 7159 & $21: 36: 54.58+57: 31: 50.2$ & 17.2580 .108 & 15.7710 .074 & 15.0350 .05 & 14.0670 .076 & 13.8790 .065 & 13.740 .721 & 12.971 .429 \\
\hline 7377 & $21: 37: 10.55+57: 29: 52.9$ & 15.1070 .028 & 14.30 .033 & 14.1340 .027 & 13.850 .013 & 13.8420 .027 & 13.8490 .11 & 14.0440 .348 \\
\hline 7491 & $21: 36: 51.54+57: 32: 53.4$ & 16.2310 .046 & 15.2850 .055 & 14.8460 .047 & 13.9170 .092 & 13.9360 .072 & 13.1720 .754 & 11.1220 .534 \\
\hline 7493 & $21: 36: 48.84+57: 33: 17.4$ & 17.5420 .122 & 16.6930 .154 & 16.5840 .21 & 14.6660 .086 & 14.9310 .087 & $\ldots$ & $\ldots$ \\
\hline 7532 & $21: 36: 54.66+57: 32: 29.4$ & $17.35^{\mathrm{c}}-$ & 16.1770 .105 & 15.270 .08 & 13.8580 .075 & 13.850 .052 & 11.820 .127 & 9.940 .124 \\
\hline 7949 & $21: 37: 00.27+57: 32: 23.8$ & $\ldots$ & $\ldots$ & $\ldots$ & 14.2560 .256 & 14.8740 .341 & 11.4540 .127 & 9.4570 .129 \\
\hline 8151 & $21: 36: 45.97+57: 34: 55.1$ & 15.1020 .047 & 14.4340 .059 & 14.2540 .036 & 13.8110 .032 & 13.9490 .061 & 14.2210 .22 & $\ldots$ \\
\hline 8183 & $21: 36: 59.85+57: 32: 56.2$ & 15.6360 .037 & 15.230 .049 & 15.0030 .053 & 14.8430 .048 & 14.6860 .049 & 14.550 .323 & $\ldots$ \\
\hline 8507 & $21: 37: 06.49+57: 32: 32.1$ & 13.330 .02 & 12.3350 .028 & 11.9040 .016 & 10.8620 .0030 & 10.5390 .0030 & 10.2010 .0070 & 9.5270 .009 \\
\hline 8689 & $21: 37: 09.39+57: 32: 25.4$ & 15.820 .116 & 15.2050 .181 & 15.0160 .07 & 13.7930 .052 & 13.3940 .091 & 13.2990 .135 & $\ldots$ \\
\hline
\end{tabular}

Notes.

a Internal ID for each object.

b Photometry for the $J, H$, and $K_{s}$ bandpasses has been taken from the 2MASS database (Cutri et al. 2003).

c Upper limits.

or II, there is a preference for the large amplitude variables to be among the evolutionarily youngest stars in our sample. In addition, if we compare the whole sample of YSOs with the group of objects showing periodic-like variations the magnitude distributions are very similar and there is no evidence that the latter ones are more or less massive than the whole sample.

Carpenter et al. (2001) presented a near-IR variability study toward a several square degree area in the Orion A molecular cloud. They found more than a thousand variable stars with a high diversity of photometric behavior. As in our case, most of their variable objects did not show color variations and $18 \%$ of them were periodic. They concluded that most of the periodic stars were better explained by cool spot models, primarily because of the small change in color during the variability cycle. Most of the periodic stars were compatible with being WTTs, based on their colors. However, some aspects of the $J H K$ data seemed incompatible with either cool or hot spot models. The small number of objects in our sample, the difference in the sample construction (in particular, the lack of Class III sources in our sample), and the different wavelength coverages prevent us from making any direct statistical comparison with the Carpenter et al. data. In addition, the thermal emission from dust at IRAC wavelengths adds a significant complication which makes it more difficult for the 

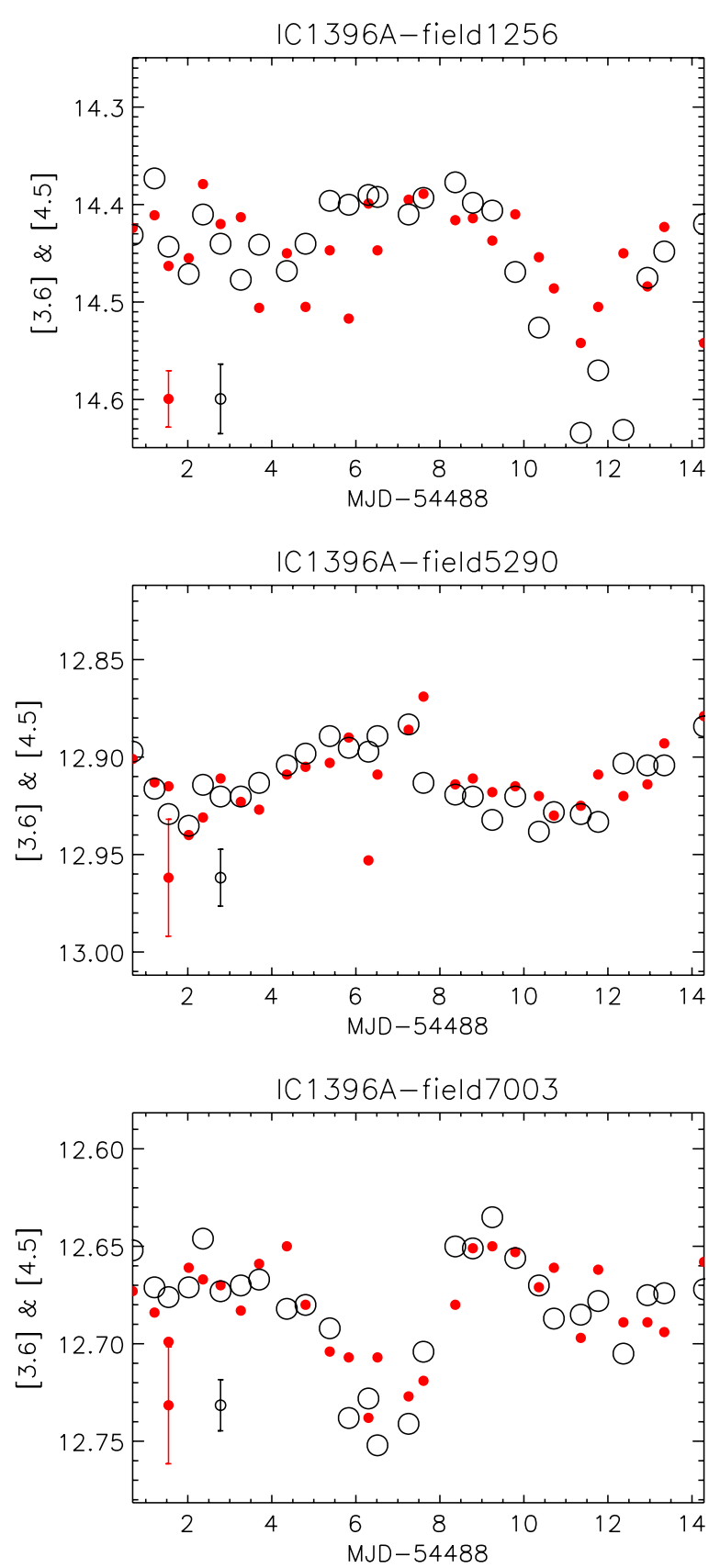

Figure 15. Light curves for a few variable candidate members of IC1396A from IRAC time-series observations. Red solid circles stand for the IRAC photometry at Ch. $1(3.6 \mu \mathrm{m})$ while black open circles represent IRAC photometry at Ch. $2(4.5 \mu \mathrm{m})$. The $4.5 \mu \mathrm{m}$ light curve has been shifted in the $y$-direction so that the mean magnitude is the same as for the $3.6 \mu \mathrm{m}$ points. Ch. 3 and Ch. 4 light curves (not shown) show the same trends as in this plot but they are noisier. (A color version of this figure is available in the online journal.)

simplest spot models to fit our data, as we discuss in the next section.

\section{CAUSES OF VARIABILITY}

\subsection{Accretion Variation}

We have investigated the possibility that the variation seen in some of our light curves come from changes in the disk mass accretion rate. D'Alessio et al. (2005) have constructed a database of accretion disks models. Using these models we have computed the amplitudes of the variability due to changes

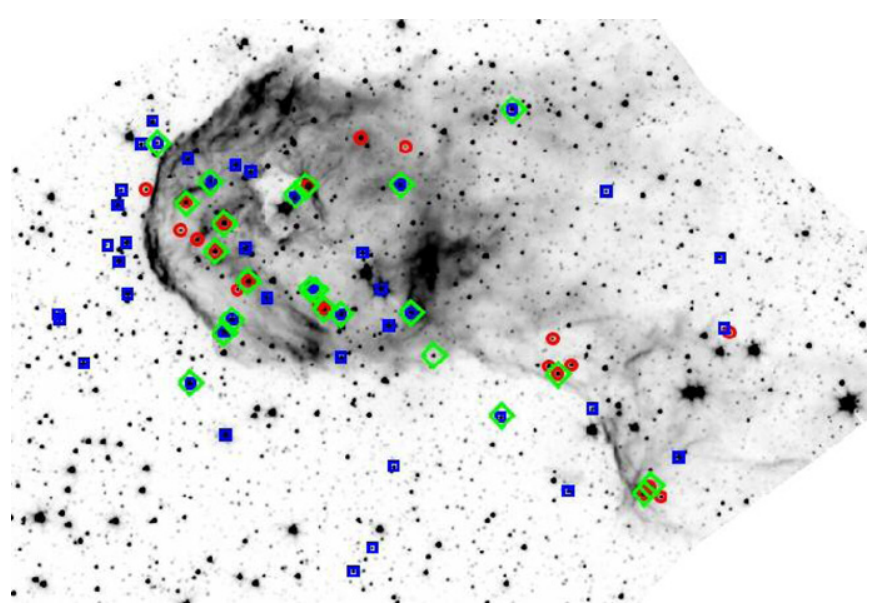

Figure 16. Spatial distribution of YSOs in IC1396A. Red circles represent the Class I objects, blue squares show the Class II objects, and stars with periodiclooking are plotted with green large diamonds.

(A color version of this figure is available in the online journal.)

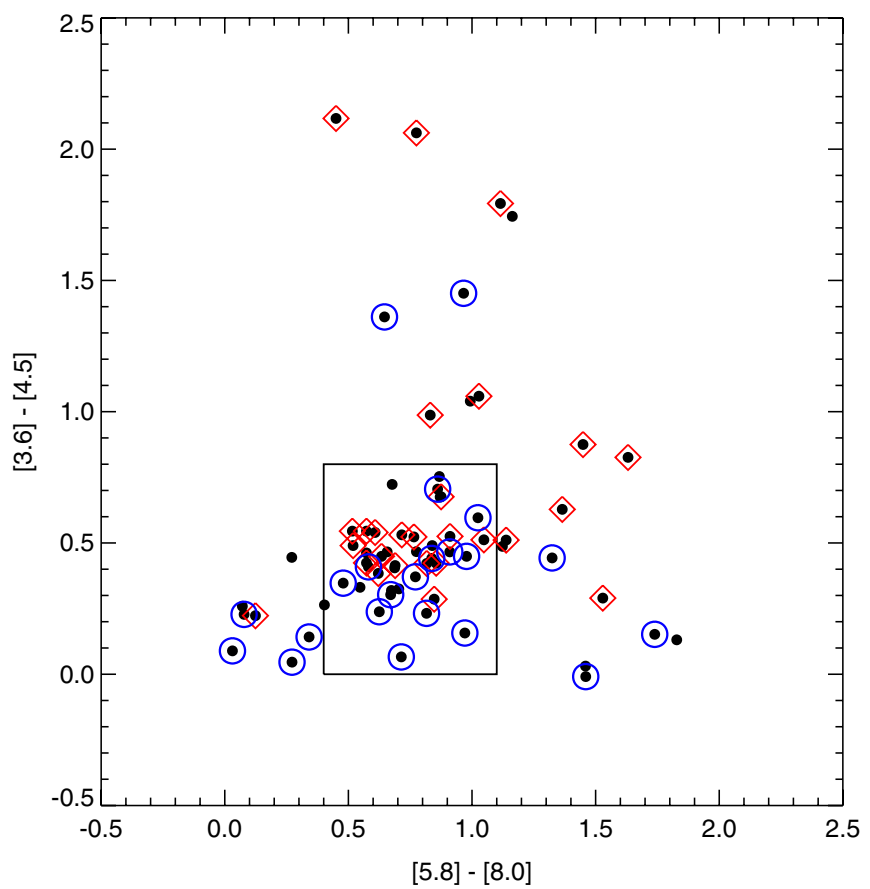

Figure 17. IRAC color-color diagram. Red open diamonds represent objects with variability amplitudes larger than $0.1 \mathrm{mag}$ and blue circles represent constant, or almost constant YSOs.

(A color version of this figure is available in the online journal.)

Table 7

Statistics for the Long-term Variability

\begin{tabular}{lcc}
\hline \hline \multicolumn{1}{c}{ Sample } & Number of Objects & \% Total $^{\mathrm{a}}$ \\
\hline Class I in the sample & 20 & 29 \\
Class II in the sample & 45 & 65 \\
Variable objects & 41 & 59 \\
Class I with amplitudes $>0.05 \mathrm{mag}$ & 15 & 22 \\
Class II with amplitudes $>0.05 \mathrm{mag}$ & 26 & 38 \\
Class I with amplitudes $>0.1 \mathrm{mag}$ & 11 & 16 \\
Class II with amplitudes $>0.1 \mathrm{mag}$ & 17 & 25 \\
Periodic-like Class I & 9 & 13 \\
Periodic-like Class II & 14 & 20 \\
Color variables & 3 & 4 \\
\hline
\end{tabular}

Note.

a Percentage to the total sample ( 69 objects). 


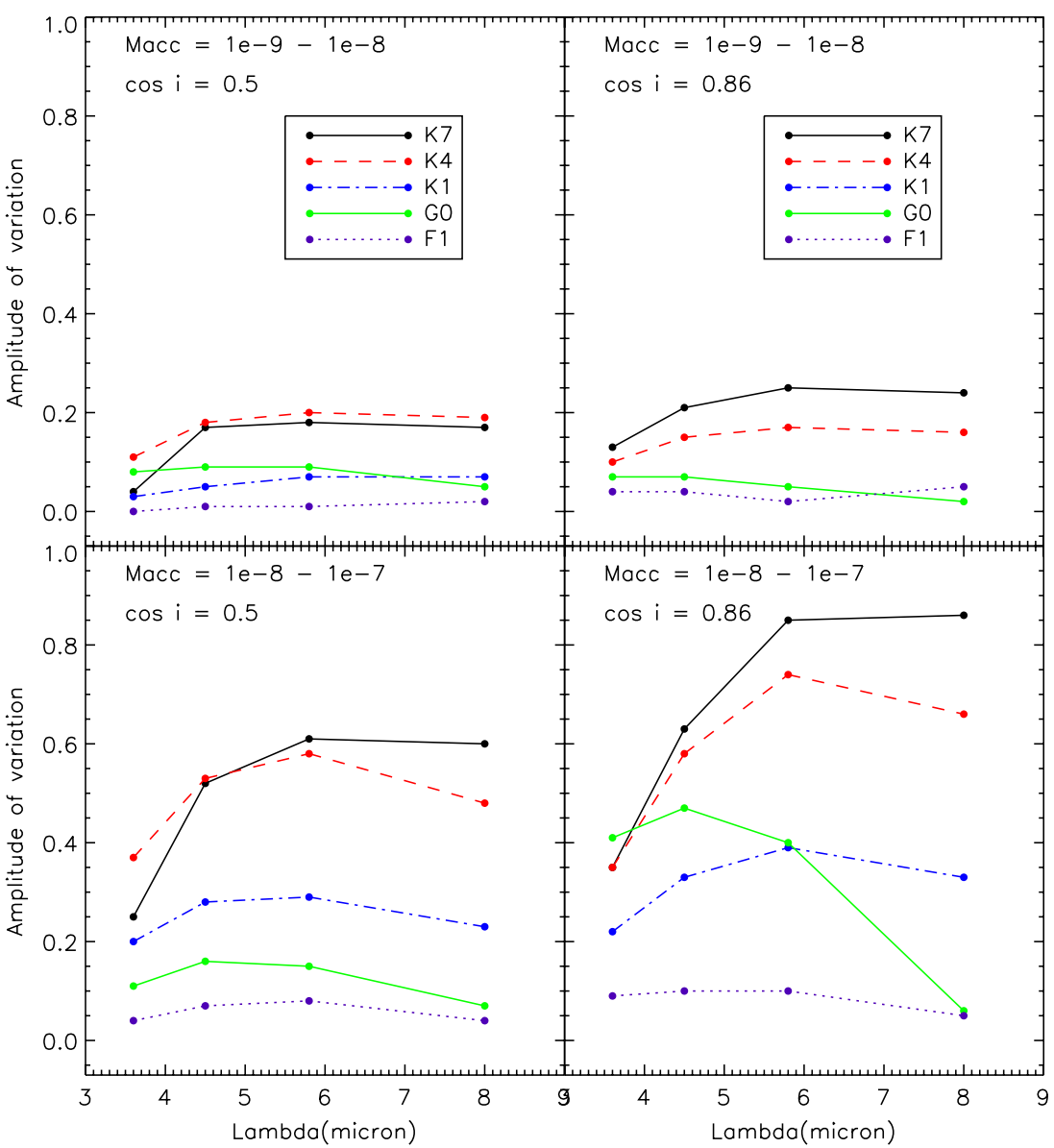

Figure 18. Amplitudes predicted by the disk models for a change in the mass accretion rate from $10^{-9} M_{\odot} \mathrm{yr}^{-1}$ up to $10^{-8} M_{\odot} \mathrm{yr}^{-1}$ in the upper panels and from $10^{-8} M_{\odot} \mathrm{yr}^{-1}$ to $10^{-7} M_{\odot} \mathrm{yr}^{-1}$ in the bottom ones.

(A color version of this figure is available in the online journal.)

of 1 order of magnitude in the mass accretion rates, from $10^{-9} M_{\odot} \mathrm{yr}^{-1}$ to $10^{-8} M_{\odot} \mathrm{yr}^{-1}$ and from $10^{-8} M_{\odot} \mathrm{yr}^{-1}$ up to $10^{-7} M_{\odot} \mathrm{yr}^{-1}$. Figure 18 shows the predicted brightness variations computed for two different variations in the accretion rates and two different inclination angles. According to these models, 1 order of magnitude change in an accretion rate $\sim 10^{-9} M_{\odot} \mathrm{yr}^{-1}$ could produce the amplitudes we see in our light curves particularly if the underlying star is relatively late spectral type. At least for some parameter ranges, the models also predict relatively little variation in IRAC color as a result of the change in accretion rate, as necessary to match most of our variable objects. This mechanism can be causing the nonperiodic variability changes on multi-day to week timescales seen in some of our variable YSOs. However, we believe that it is not the predominant mechanism; such rapid, extreme accretion variability would be easily detected at shorter wavelengths, yet few examples have been reported.

\subsection{Starspots}

More than half of the IC1396A variable YSOs have light curves that appear periodic, as shown in Figure 8, with amplitudes $0.1-0.2 \mathrm{mag}$ (see Table 4), and typical periods in the range 5 to more than 11 days. For T Tauri stars observed in the optical or near-IR such variability is thought to originate mainly from either cool magnetic spots (dynamo-driven activity) or hot accretion spots on the stellar surface (where the material from the disk falls onto the star) that are hundreds to thousands of kelvins different in temperature from the photosphere and rotate with the star. Light-curve amplitudes in the optical of the order of $0.2 \mathrm{mag}$ (or considerably larger) are not atypical for CTTs or WTTs. We have contemplated the possibility that hot or cool spots on the photospheres of these YSOs could produce the light curves we observe; using somewhat extreme parameters, lightcurve amplitudes of the order of $0.2 \mathrm{mag}$ can be obtained even at IRAC wavelengths. We have estimated the photometric amplitudes expected from both cool and hot starspots using a simple model (Vrba et al. 1986) which assumes that the stellar surface has a spotted region characterized by a single temperature blackbody and is confined to one hemisphere. The amplitude of the light variations as a function of wavelength can be expressed as

$$
m(\lambda)=-2.5 \log \left\{1-f\left[1-B_{\lambda}\left(T_{\text {spot }}\right) / B_{\lambda}\left(T_{*}\right]\right\},\right.
$$

where $T_{\mathrm{spot}}$ and $T_{*}$ are the temperatures of the spot and the photosphere, $f$ is the maximum fraction of the stellar photosphere covered by spots, and $B_{\lambda}(T)$ is the Planck function. Note that this starspot model is an approximation since it ignores limb darkening, inclination effects, and opacity differences.

Figure 19 shows the maximum predicted amplitudes at $3.6 \mu \mathrm{m}$ and color [3.6]-[4.5] for typical stars with temperatures ranging between $3000 \mathrm{~K}$ and $6000 \mathrm{~K}$ (expected temperatures for Two Micron All Sky Survey (2MASS) $J$ magnitudes between 9.5 and 14 mag at $900 \mathrm{pc}$ if one assumes an age of $0.5 \mathrm{Myr}$; Siess et al. 2000). We assumed that hot spots (filled circles) have temperatures of the order of $10,000 \mathrm{~K}$, and cold spots 


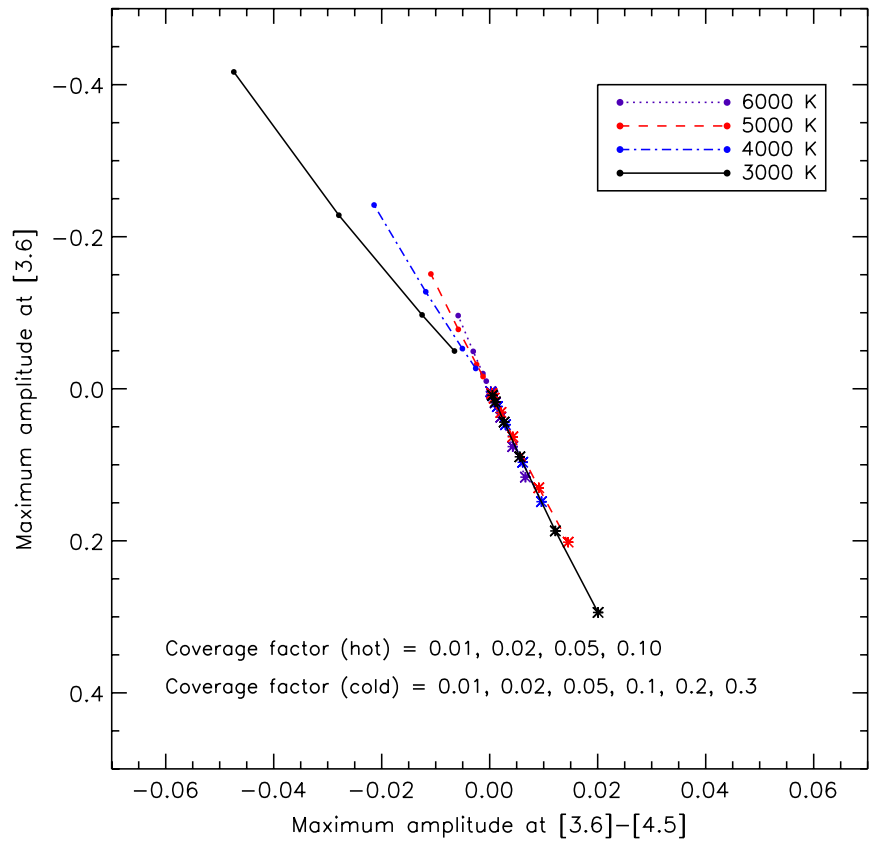

Figure 19. Maximum amplitudes predicted by the spot model for a set of stars with temperatures ranging from $3000 \mathrm{~K}$ to $6000 \mathrm{~K}$. Filled circles and asterisks represent the amplitudes predicted for hot and cold spots, respectively.

(A color version of this figure is available in the online journal.)

(represented by asterisks) have temperatures $1500 \mathrm{~K}$ cooler than the photosphere. Different coverage fractions with a maximum of $10 \%$ for hot spots, and up to $30 \%$ for cold spots are shown. In Figure 20, the maximum amplitude of variation for each channel is shown. These diagrams show that the difference in amplitude between different bandpasses is very small, as is true for our light curves, and the amplitudes are smaller for higher photospheric temperatures.

However, these simple spot models are missing an important contribution relevant to our case. Essentially all of our objects are Class I and Class II YSOs, with significant flux excesses over photospheric at IRAC wavelengths. The Vrba et al. (1986) model assumes that all of the flux is coming from photosphere and spots. If warm dust in the disk or envelope contribute substantially to the integrated fluxes at $3.6 \mu \mathrm{m}$ onward, and if the disk/envelope flux is invariant with time, then the variability amplitudes we observe with IRAC would be substantially lower than predicted by Equation (2).

We consider two extreme cases. At one extreme, we consider the case where nearly all of the light we see from the variable YSOs is scattered light. The apparent excess fluxes at IRAC wavelengths are therefore instead indicative of lower extinction to the scattering surface at longer wavelengths, rather than thermal emission from dust in the inner disk/envelope. Since all of the light we see originates from the star, the IRAC light-curve amplitudes could be nearly as large as the model estimates, dependent on the geometry assumed for the scattering surface(s). Wood \& Whitney (1998) have constructed models of Class I YSOs with hot spots illuminating a disk+envelope, and have shown that even when the direct light from the star is heavily attenuated, the photometric signature of the hot spot (as it rotates with the stellar photosphere) can be seen in the scattered light flux from the envelope, at least at the $I$ band. For a model where the star+hot spot had an I-band light-curve amplitude of around 1 mag and where direct light from the star was almost completely blocked by the disk ( $i=82 \mathrm{deg}$ ), the scattered light at the $I$ band
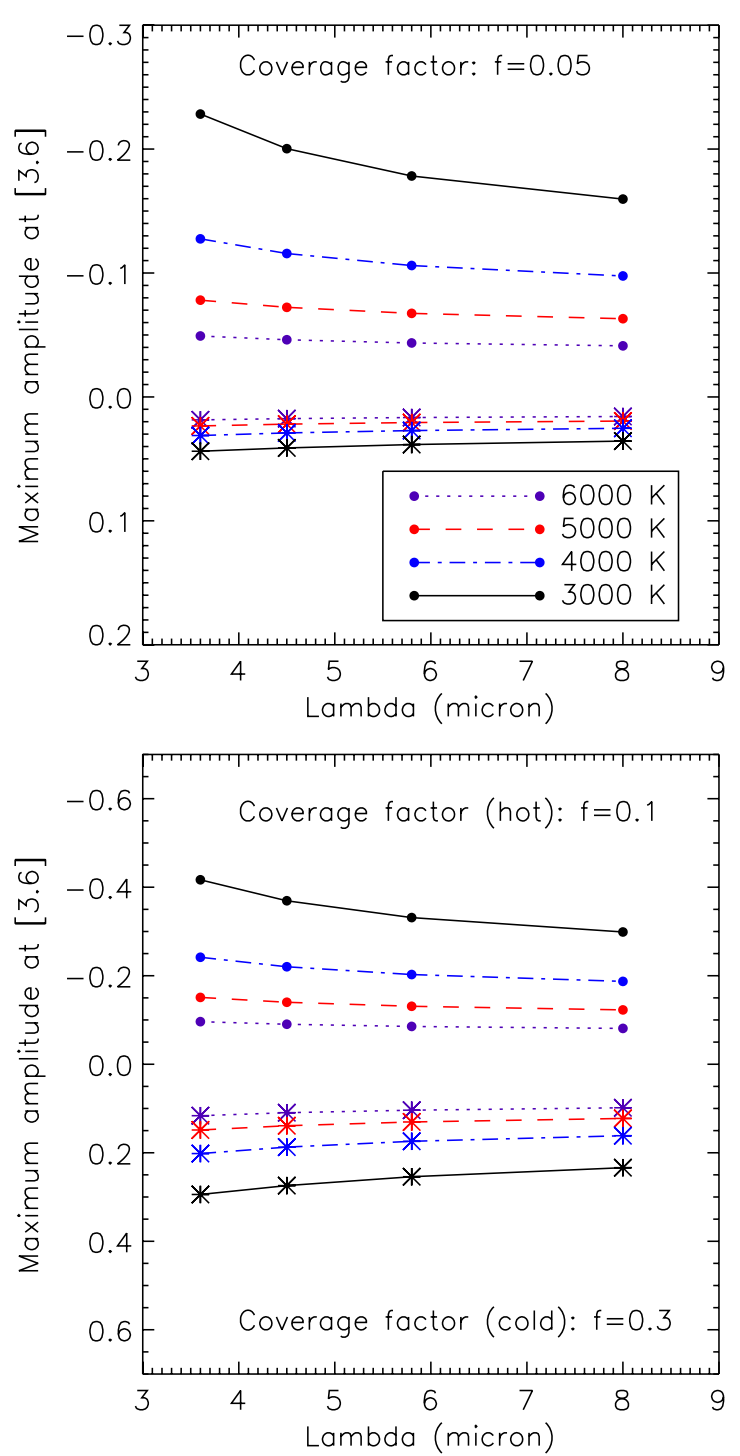

Figure 20. Maximum amplitudes predicted by the spot model for a set of stars with temperatures ranging from $3000 \mathrm{~K}$ to $6000 \mathrm{~K}$. Filled circles and asterisks represent the amplitudes predicted for hot and cold spots, respectively. The upper panel shows intermediate coverage factor while the bottom panel presents a more extreme situation with a coverage factor of 0.1 for hot spots and a coverage factor of 0.3 for cold spots.

(A color version of this figure is available in the online journal.)

showed a light-curve amplitude of about $0.2 \mathrm{mag}$. A possible prototype for our IC1396A variable objects is HL Tau, where it is believed that essentially all the flux we see at least out to $2 \mu \mathrm{m}$ is scattered light. If scattered light dominates out to $8 \mu \mathrm{m}$, then perhaps the Wood \& Whitney (1998) model could provide the correct physical model to explain our light curves. With these assumptions, the observed flux at IRAC Ch. 4 provides a lower limit to the photospheric flux of the star at $8 \mu \mathrm{m}$. For our stars with quasi-sinusoidal light curves, and assuming an age of 0.5 Myr from Siess et al. (2000), this suggests masses of the order of $0.3-4.5 M_{\odot}$, and spectral types of M5 to F3 with the majority of the objects having masses around $3.5 M_{\odot}$ and spectral types $\sim \mathrm{K} 3$.

However, it is not obvious that the variable YSOs in IC1396A are as evolutionarily young as HL Tau. Some of them fall in the IRAC color-color diagram within the Class II region, and do not necessarily have any significant remaining infalling envelope to 
serve as the scattering surface. ${ }^{7}$ Also, it is difficult to understand how these stars could have such extreme hot spots (much larger covering factor than typically required to explain CTT light curves in the optical or near-IR) and hence presumably very active accretion without having a significant amount of warm dust contributing strongly to the flux at IRAC wavelengths. Therefore, as the other extreme model, we consider a case where the observed $J$-band magnitude is assumed to provide a reasonable estimate for the photospheric flux of the YSO (an assumption often used when attempting to place YSOs in $\mathrm{H}-\mathrm{R}$ diagrams to estimate their ages). The fluxes at IRAC wavelengths are then the sum of a photospheric component (estimated by taking the $J$-band flux and extrapolating to longer wavelengths with a blackbody function) plus excess emission from warm dust. The fraction of the IRAC flux that is emitted by the star then places constraints on models to explain the light-curve amplitudes. As an example, we derive $F_{\text {phot }}=0.09$, $0.04,0.02$, and 0.01 for IRAC Ch. 1 through Ch. 4 for object IC1396A-47 (see Figure 23), where $F_{\text {phot }}$ is the fraction of the total system flux coming from the star in each of the IRAC bands. In the absence of any other mechanisms, and assuming the disk emission is invariant with time, if the star had an amplitude in its IRAC light curves of $1 \mathrm{mag}$, the star+disk would have amplitudes of only of the order of $0.09 \mathrm{mag}$ in Ch1 and $0.01 \mathrm{mag}$ in Ch4. Because no plausible spot model produces light-curve amplitudes anywhere near 1 mag at IRAC wavelengths (for just the star alone), and because we see no wavelength-dependent variation in amplitude for objects similar to IC1396A-47, we conclude that this simplest star+spot plus disk model cannot account for our the observed light curves.

\subsection{Variable Heating of the Disk and Disk Inhomogeneities}

Time varying heating of the inner disk by the rotating spot could provide the mechanism for IRAC variability, if some property of the viewing geometry allows our view of the warmer part of the disk to vary with time. In this case, it is the luminosity of the spot compared to the luminosity of the rest of the photosphere that matters, so if the temperature of the hot spot is twice that of the photosphere, a spot covering fraction of only a little more than $1 \%$ could yield a 0.2 mag light-curve amplitude from the disk. For this flavor of models, and assuming a medium Av of $8 \mathrm{mag}$, the $J$-band fluxes correspond to a mass range of $0.2-1.3$ solar mass for our periodic YSOs.

We have constructed models of a Class I protostar (with a disk and envelope, and a photosphere with a hot spot) using the radiative transfer code described in Whitney et al. (2003a, 2003b), and have varied the inputs to these models, including the inclination angle at which we view the disk. If there are hot spots in the photosphere of the object, the disk will be asymmetrically heated too, and that could help keep the variations at IRAC bands at a higher level than if the disk emission were a constant source of additional flux. Figure 21 shows the results of these models for two viewing angles, with the temporal variation of the SED in the left panels, and the magnitude variations in specific bands for one full rotation period of the star in the right panels (asterisk: $V$ band, diamond: $R$ band, triangle: [3.6], box: [4.5], open circle: [5.8], and filled circle: [8.0]). A star of mass $1.5 M_{\odot}$ with effective temperature $\sim 4300 \mathrm{~K}$ was used.

\footnotetext{
7 We note, however, that according to the models of Robitaille et al. (2007), some Class I YSOs have colors that fall in the Class II region of the IRAC $\mathrm{CCD}$, and therefore it is possible that all of our "periodic" variables may be Class I's.
}

For accretion parameters, we used a disk mass of $0.05 M_{\odot}$, a disk accretion rate of $8.6 \times 10^{-7} M_{\odot} \mathrm{yr}^{-1}$, and an envelope accretion rate of $4 \times 10^{-6} M_{\odot} \mathrm{yr}^{-1}$. A hot spot with a fractional area coverage of $0.3 \%$ and a temperature of $10,000 \mathrm{~K}$ sitting at latitude 45 deg was added to the models. The figure shows that these models can easily reproduce the IRAC light curves with the right amplitudes with very modest hot spot sizes.

Close examination of the details of the model shows that the IRAC variations arise from the heating of the inner disk wall (that is, the IRAC light curves are brightest when the photospheric hot spot is on the far side of the star, heating up the back wall). The IRAC variations are largest at close-toedge-on viewing angles where the far side of the wall is in view. At more pole-on inclinations (bottom panels of Figure 21), the flux from the inner disk shows less phase dependence of our view of the hotter part of the inner disk so contribute less to the overall flux which consequently shows smaller variations. In the optical, the star is brightest when the hot spot is facing the observer. Therefore, the optical and IRAC light curves are 180 deg out of phase.

Similar results are obtained for a Class II source as shown in Figure 22. The top-left panel shows the same stellar, disk, and hot spot parameters as in Figure 21 but with no envelope. The IRAC variations are similar (the $y$-axis scales are different), but the visible light curve shows much larger variation in the Class II source. In the Class I source, the visible light is scattered in the envelope, which decreases and washes out the variations. In the Class II source, we clearly see the stellar hot spot rotate out of view and then stay at the same brightness until it rotates into view.

The top right panel of Figure 22 adds a warp to the disk: at the same longitude as the stellar hot spot, the disk height increases by $25 \%$ over the nominal value (we use a $\sin ^{11}(\Lambda)$ function, where $\Lambda$ is the longitude, to make a smooth transition). The warp decays exponentially with radius, with an $e$-folding distance equal to the inner disk radius $R_{\text {in }}$; thus, at a radius of several $R_{\text {in }}$, the disk becomes axisymmetric. The warp is meant to simulate the effect of an accretion flow to the stellar hot spot, and dragging some dust with it, effectively increasing the height of the disk. A model of this type has previously been proposed to explain the observed optical variability of AA Tau (Bouvier et al. 1997, 2003).

The result of this is that the IRAC variations are larger except at $8 \mu \mathrm{m}$. This is because the regions of the wall that is heated by the stellar hot spot have a bigger area than the model with no warp. The optical variations are lower with the inclusion of the warp. This is because the reflection off the warped wall adds flux back into the beam as the hot spot moves out of view.

The bottom panels of Figure 22 show two stellar hot spots separated in longitude by $180^{\circ}$, with one at $+45^{\circ}$ latitude and the other at $-45^{\circ}$, and disk warps at these longitudes (one in the $+z$ direction and the other in the $-z$ direction). These are shown for two different viewing angles (bottom left and right panels). The IRAC light curves are now double peaked due to the two warps. The IRAC variations are similar in the two viewing angles shown (bottom left and right panels), while the visible light curves show large differences. The optical light curves are more complicated due to the geometry of the stellar hot spots, and scattering and obscuration from the warped wall.

These models demonstrate that both hot spots and disk inhomogeneities can explain the variability in the $30 \%$ of our sample of YSOs that shows 5-12 day periodic light curves. The amplitudes and behaviors are a function of the spot temperature 

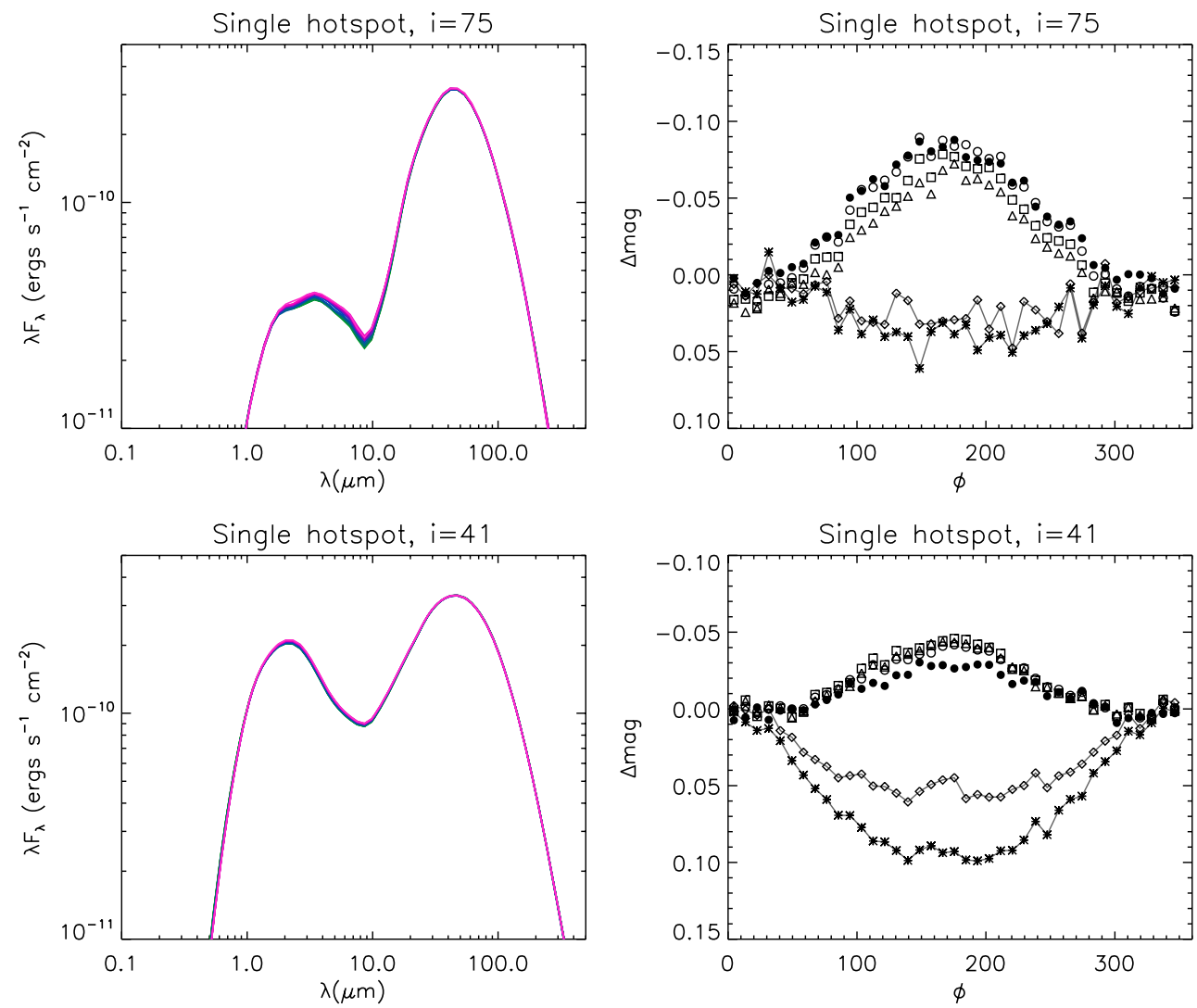

Figure 21. Model results for a Class I object with a hot spot on the photosphere, at a latitude of $45^{\circ}$. The different color SEDs (left) correspond to different phase angles; and the light curves (right) show one full rotation period at various wavebands (asterisk: $V$ band, diamond: $R$ band, triangle: [3.6], box: [4.5], open circle: [5.8], and filled circle: [8.0]). The $V$ - and $R$-band light curves are connected by lines to distinguish them from the IR light curves. Phase 0 corresponds to the stellar hot spot facing the observer. At phase $=180 \mathrm{deg}$, the hot spot is obscured and the heated far wall faces the observer. Positive $\Delta$ mag corresponds to fainter fluxes. The visible light curves are noisier and washed out due to extinction and scattering from the envelope. The results are periodic colorless IR variations with amplitudes similar to those that we observe at IRAC bands, and with the brightest optical phase corresponding to the faintest IRAC phase. At more pole-on viewing angles (bottom panels), the visible light curves show more variation, and the IR light curves show less. This is because the stellar hot spot, located at 45 deg latitude, shows more pronounced variation with rotation, whereas the flux from the inner disk shows less phase dependence with more pole-on viewing angle.

(A color version of this figure is available in the online journal.)

and size, number of hot spots, viewing angle, and possibly inner disk radius (the latter has not been investigated yet). Multiwavelength observations combined with models should be able to distinguish between single- and double-hot spot models and viewing angles.

\subsection{IC1396A-47: Pulsating Pre-Main-Sequence Star?}

Our object IC1396A-47 was previously identified in Reach et al. (2004) as object $\zeta$. It has been classified by us and others as a Class I YSO based on its IRAC SED shape and position in the IRAC CCD. However, when we fit its SED using an evolutionary sequence of models from Class 0 to Class III (Whitney et al. 2003a, 2003b), object 47 is best fit with a pure disk model seen nearly edge on, with essentially no envelope, and with a large extinction to explain the very red $J-K$ color. The primary reason why an edge-on Class II model is favored is the low $24 \mu \mathrm{m}$ flux.

IC1396A-47 shows a periodic variation with a peak-to-peak amplitude of $0.2 \mathrm{mag}$ and a derived period of 9 days in the mapping data (see Figure 23(b)). However, this object was the only one also showing a periodic short-term variation. As shown in Figure 23(a), it exhibits an apparent $\sim 3.5 \mathrm{hr}$ periodicity with amplitude of the order of 0.04 mag. Contact binaries can have similar periods and light-curve shapes; however, for the mass and radius required for object zeta, the apparent period is too short. We believe that the most plausible explanation for the short-period variability is that object zeta is a PMS delta Scuti (pulsational) variable. A few PMS $\delta$ Scuti pulsators have been identified and some new possible members have also been proposed (Rodríguez \& Breger 2001; Zwintz et al. 2005; Kallinger et al. 2008). Breger (1972) identified two PMS $\delta$ Scuti variables in NGC 2264-both have colors of F stars, periods of the order of $3 \mathrm{hr}$ and $V$-band light-curve amplitudes of the order of $0.05 \mathrm{mag}$.

If the $J$-band flux of IC1396A-47 were photospheric, an $A(V)$ of 50 mag would be required to allow zeta to have the minimum mass to fall within the instability strip at $0.5 \mathrm{Myr}$ (Marconi $\&$ Palla 1998). If the Ch.4 flux is purely photospheric (see Section 6), the derived mass for this object is $\sim 4-4.5 M_{\odot}$ (spectral type F3 and implied Av = 35) which is compatible with it being in the instability strip.

\section{SUMMARY AND CONCLUSIONS}

We have conducted a variability study using Spitzer/IRAC data keeping in mind that mid-IR variability probes different physical mechanisms than variability in the optical or nearIR. Because IRAC is sensitive to the dust in the inner disk and envelope, variability at IRAC wavelengths in YSOs should primarily trace changes in the heating and fluctuations in the structure of the circumstellar disk and envelope surrounding the nascent star whereas optical and near-IR variability is directly 

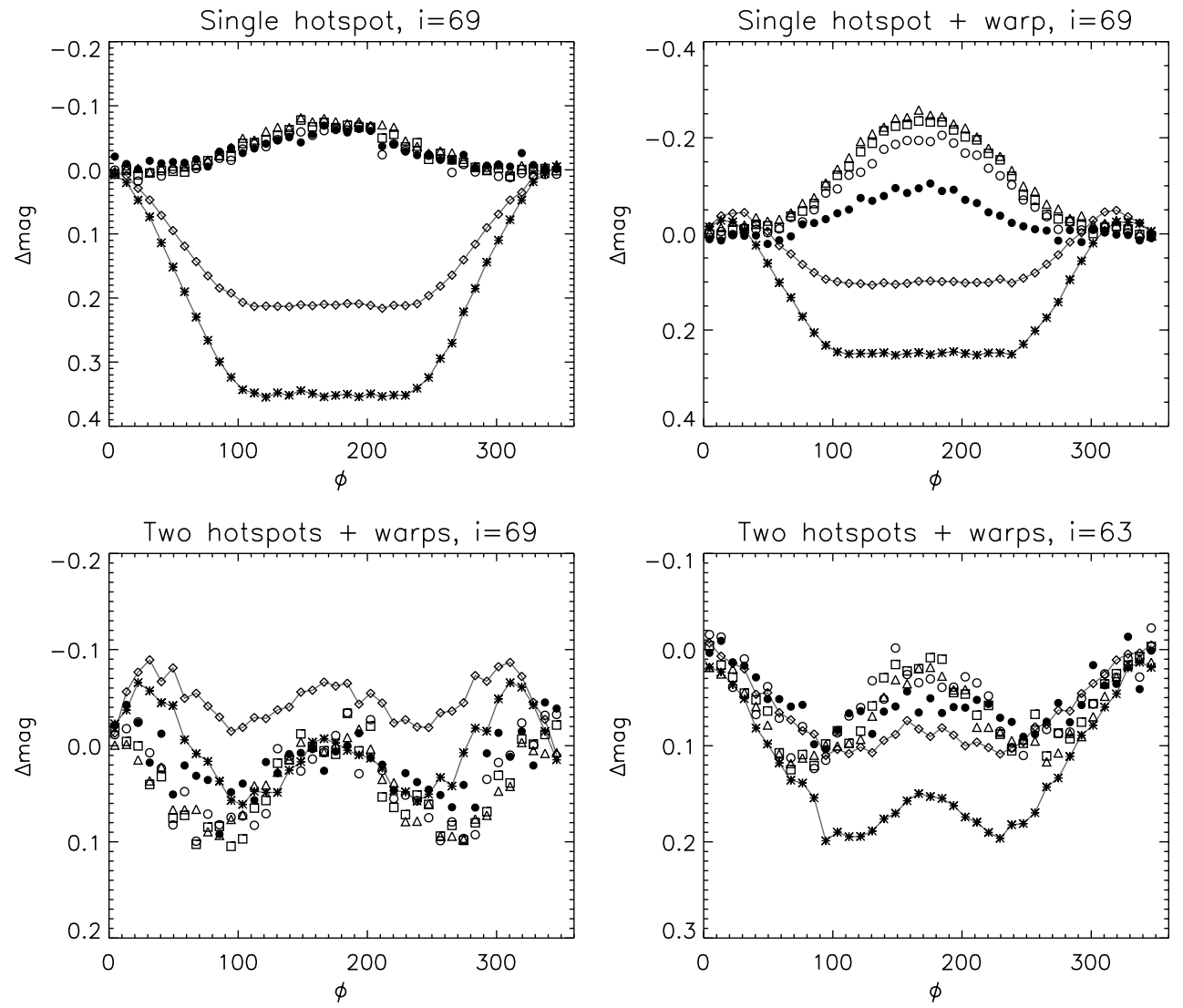

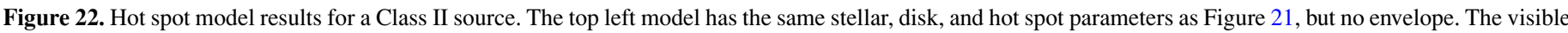

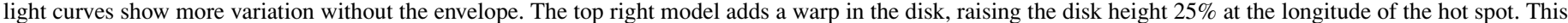

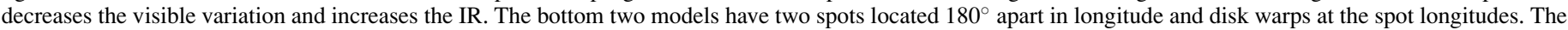
visible light curves vary more with inclination (bottom left and bottom right). The symbols are as in the previous figure.

related to the central object. Detailed IRAC light curves thus have the potential to allow us to construct models of the structure of the inner disk and the temporal variations in disk structure and mass accretion. Some of these variations could be tied to either the formation or migration of planets (Ida \& Lin 2008). Therefore, mid-IR variability offers a new means to infer how stars are assembled and the possible early stages of planet formation.

Our photometric monitoring program on the IC1396A dark globule focuses on studying the mid-IR variability of the heavily embedded YSOs present in that area. Observations were performed with Spitzer/IRAC using two different setups corresponding to distinct timespan and temporal cadences: (1) light curves covering a 14 day timespan with a twice daily cadence for 69 YSOs, and (2) continuous light curves with approximately $12 \mathrm{~s}$ cadence over $7 \mathrm{hr}$ for 38 YSOs. Typical accuracies for our relative photometry were of the order of $3 \mathrm{mmag}$ for the short-term data, and around $10 \mathrm{mmag}$ for the longer-term data. This is the only star-forming region for which such extensive time-series photometry has been obtained with Spitzer.

Around $60 \%$ of our sample of YSOs (69 objects) were determined to be variable on long timescales and eight objects (out of the 38 objects with continuous light curves over a $7 \mathrm{hr}$ timespan) are also labeled as variable in short timescales. We have found very different shapes in the long-term light curves but the most noticeable characteristic is the very good similarity among the light curves in different bandpasses both in shape and in the amplitude of the variation. About $30 \%$ of our total sample of YSOs show periodic-like, colorless variability with amplitudes up to about $0.2 \mathrm{mag}$. These quasi-periodic objects are about equally divided between Class I and Class II and have possible masses ranging from 0.2 to $\sim 4.0 M_{\odot}$ (depending on the assumed extinction). We have investigated different scenarios to understand the cause of this type of variability, variable accretion, hot and cold spots in the photosphere of the objects, and variable obscuration produced by the circumstellar disk. We believe that the most plausible cause of variability for these objects are hot spots on the photosphere of our targets which asymmetrically heat the inner wall of the disk (Figure 21). Disk warping can enhance this effect (Figure 22). We have used radiation transfer models to check this scenario and the models are able to reproduce the observed variability in the four IRAC bands with only small changes in accretion and/or inclination angle needed to get the range of amplitudes found in our YSOs.

One of our targets, IC1396A-47, was the only one also showing periodic short-term variation with an apparent $\sim 3.5 \mathrm{hr}$ periodicity and an amplitude of the order of 0.04 mag. We propose that IC1396A-47 is a PMS delta Scuti variable. A dedicated, long duration photometric monitoring campaign on this object could in principle determine its mass and age from its pulsation frequencies, thereby testing theoretical PMS models at very young ages.

In addition to the variability study we have searched for new IC1396A candidate members using two different methodologies. On the one hand, we co-added the observations to obtain a deeper mosaic and extracted 15 new candidate members, 13 out of them fall into the Class II region in the IRAC color-color 

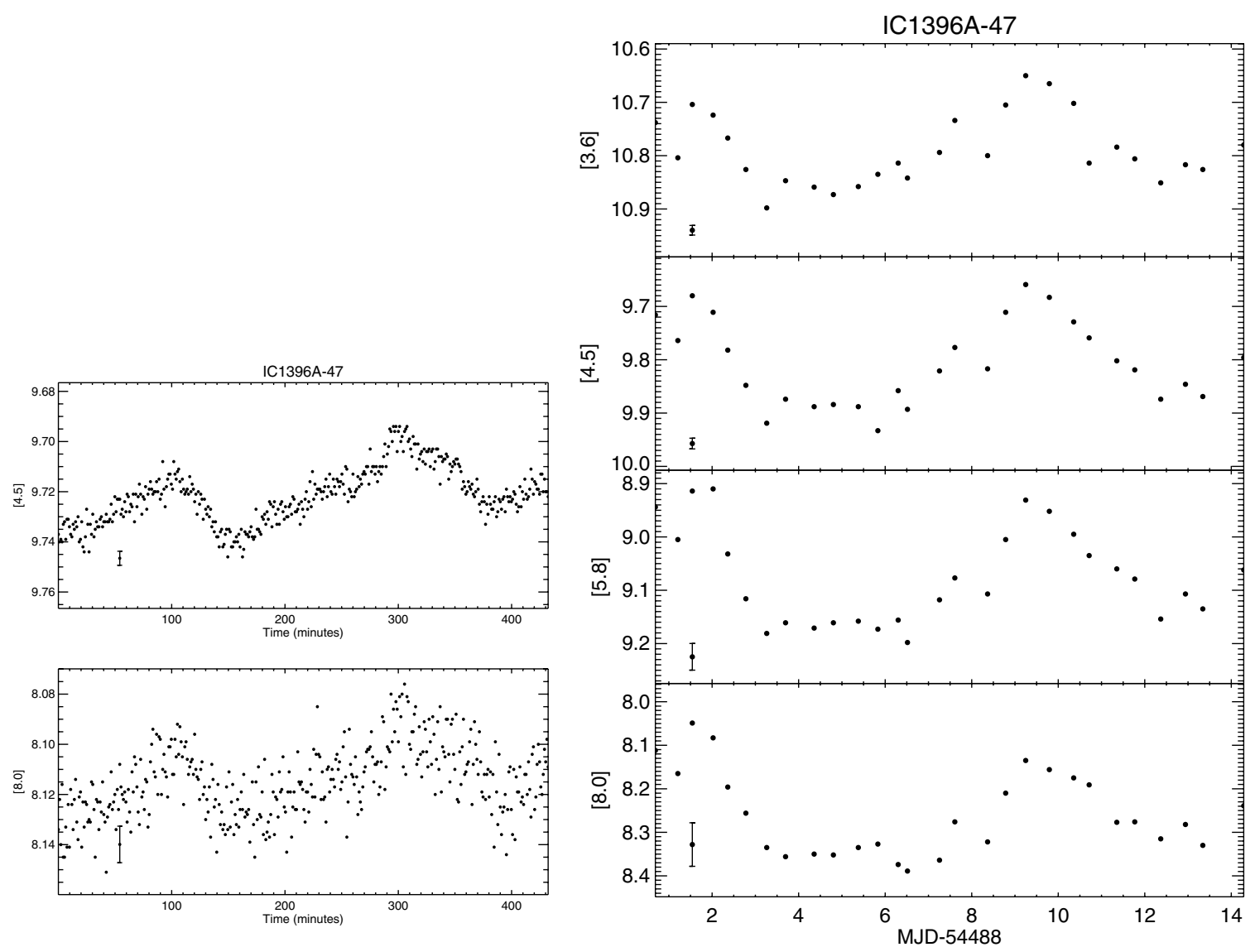

Figure 23. (a) Light curves of IC1396A-47 (object $\zeta$ ) showing the $3.5 \mathrm{hr}$ period variation from the $7 \mathrm{hr}$ staring-mode IRAC observation. The top and bottom panels show Ch. 2 and Ch. 4 time series, respectively. (b) Light curves of the same object from IRAC mapping observations showing the $\sim 9$ days period variation in the four IRAC bandpasses.

diagram and two are classified as Class I objects. On the other hand, we have searched for variability, as an indicator of youth, in the field and have detected around 50 possible new Class III candidates.

These observations have shown that Spitzer/IRAC is an exquisite instrument to carry out very accurate photometric monitorings both in timescales of hours and days. During the post-cryogenic operations, it will be the prime means to carry out this type of studies until the arrival of the James Webb Space Telescope.

This work is based [in part] on observations made with the Spitzer Space Telescope, which is operated by the Jet Propulsion Laboratory, California Institute of Technology under a contract with NASA. This research has been funded by the NASA grant JPL101185-07.E.7991.020.6, and the Spanish grants MEC/ESP 2007-65475-C02-02, MEC/ConsoliderCSD2006-0070, and CAM/PRICIT-S-0505/ESP/0361. M.M.C. acknowledges the support by a predoctoral "Calvo Rodeés" fellowship by INTA.

Facilities: Spitzer (IRAC)

\section{REFERENCES}

Allen, L. E., et al. 2004, ApJS, 154, 363

Bouvier, J., Bertout, C., \& Bouchet, P. 1986, A\&A, 158, 149

Bouvier, J., Forestini, M., \& Allain, S. 1997, A\&A, 326, 1023

Bouvier, J., et al. 2003, A\&A, 409, 169

Breger, M. 1972, ApJ, 171, 539
Byrne, P. B., Doyle, J. G., Brown, A., Linsky, J. L., \& Rodono, M. 1987, A\&A, 180,172

Carpenter, J. M., Hillenbrand, L. A., \& Skrutskie, M. F. 2001, AJ, 121, 3160

Castelli, F., Gratton, R. G., \& Kurucz, R. L. 1997, A\&A, 318, 841

Charbonneau, D., et al. 2005, ApJ, 626, 523

Contreras, M. E., et al. 2002, AJ, 124, 1585

Cutri, R. M., et al. 2003, 2MASS All Sky Catalog of Point Sources, The IRSA 2MASS All-Sky Point Source Catalog, NASA/IPAC Infrared Science Archive, http://irsa.ipac.caltech.edu/applications/Gator/

D’Alessio, P., Merín, B., Calvet, N., Hartmann, L., \& Montesinos, B. 2005 RevMexAA, 41, 61

Deming, D., et al. 2007, ApJ, 667, L199

Ida, S., \& Lin, D. N. C. 2008, ApJ, 673, 487

Kallinger, T., Zwintz, K., \& Weiss, W. 2008, A\&A, 488, 279

Lada, C. J., et al. 2006, AJ, 131, 1574

Marconi, M., \& Palla, F. 1998, ApJ, 507, L141

Megeath, S. T., et al. 2004, ApJS, 154, 367

Morales-Calderón, M., et al. 2006, ApJ, 653, 1454

Reach, W. T., et al. 2004, ApJS, 154, 385

Reach, W. T., et al. 2005, PASP, 117, 978

Robitaille, T. P., Whitney, B. A., Indebetouw, R., \& Wood, K. 2007, ApJS, 169, 328

Rodríguez, E., \& Breger, M. 2001, A\&A, 366, 178

Rydgren, A. E., \& Vrba, F. J. 1983, ApJ, 267, 191

Scargle, J. D. 1982, ApJ, 263, 835

Sicilia-Aguilar, A., et al. 2006, ApJ, 638, 897

Siess, L., Dufour, E., \& Forestini, M. 2000, A\&A, 358, 593

Stetson, P. B. 1996, PASP, 108, 851

Vrba, F. J., Rydgren, A. E., Chugainov, P. F., Shakovskaia, N. I., \& Zak, D. S. 1986, ApJ, 306, 199

Whitney, B. A., Wood, K., Bjorkman, J. E., \& Cohen, M. 2003a, ApJ, 598, 1079

Whitney, B. A., Wood, K., Bjorkman, J. E., \& Wolff, M. J. 2003b, ApJ, 591, 1049

Wood, K., \& Whitney, B. 1998, ApJ, 506, L43

Zwintz, K., Marconi, M., Reegen, P., \& Weiss, W. W. 2005, MNRAS, 357, 345 